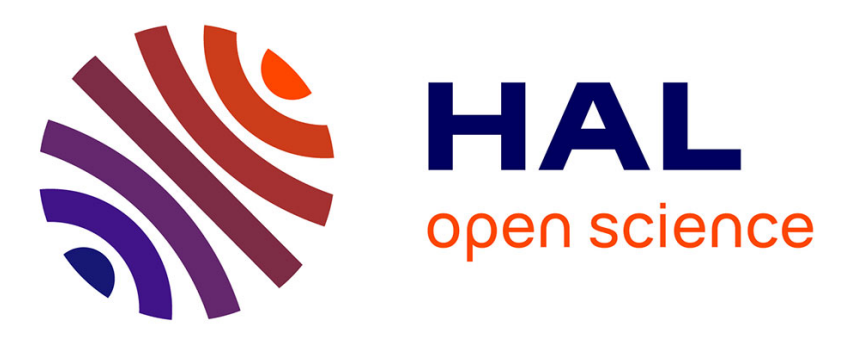

\title{
Dimensional reductions of a cardiac model for effective validation and calibration
}

Matthieu Caruel, Radomir Chabiniok, Philippe Moireau, Yves Lecarpentier, Dominique Chapelle

\section{- To cite this version:}

Matthieu Caruel, Radomir Chabiniok, Philippe Moireau, Yves Lecarpentier, Dominique Chapelle. Dimensional reductions of a cardiac model for effective validation and calibration. Biomechanics and Modeling in Mechanobiology, 2014, 13 (4), pp.897-914. 10.1007/s10237-013-0544-6 . hal-00872746

\section{HAL Id: hal-00872746 https://hal.science/hal-00872746}

Submitted on 14 Oct 2013

HAL is a multi-disciplinary open access archive for the deposit and dissemination of scientific research documents, whether they are published or not. The documents may come from teaching and research institutions in France or abroad, or from public or private research centers.
L'archive ouverte pluridisciplinaire HAL, est destinée au dépôt et à la diffusion de documents scientifiques de niveau recherche, publiés ou non, émanant des établissements d'enseignement et de recherche français ou étrangers, des laboratoires publics ou privés. 


\title{
Dimensional reductions of a cardiac model for effective validation and calibration
}

\author{
M. Caruel - R. Chabiniok - P. Moireau - Y. Lecarpentier - D. Chapelle
}

the date of receipt and acceptance should be inserted later

\begin{abstract}
Complex 3D beating heart models are now available, but their complexity makes calibration and validation very difficult tasks. We thus propose a systematic approach of deriving simplified reduced-dimensional models, in "0D" - typically, to represent a cardiac cavity, or several coupled cavities - and in "1D" - to model elongated structures such as muscle samples or myocytes. We apply this approach with an earlierproposed 3D cardiac model designed to capture lengthdependence effects in contraction, which we here complement by an additional modeling component devised to represent length-dependent relaxation. We then present experimental data produced with rat papillary muscles samples when varying preload and afterload conditions, and we achieve some detailed validations of the 1D model with these data, including for the lengthdependence effects that are accurately captured. Finally, when running simulations of the 0D model precalibrated with the 1D model parameters, we obtain pressure-volume indicators of the left ventricle in good agreement with some important features of cardiac physiology, including the so-called Frank-Starling mechanism, the End-Systolic Pressure-Volume Relationship (ESPVR), as well as varying elastance properties. This integrated multi-dimensional modeling approach thus sheds new light on the relations between the phenomena
\end{abstract}

M. Caruel, P. Moireau and D. Chapelle

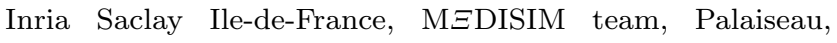
France

\section{R. Chabiniok}

Division of Imaging Sciences \& Biomedical Engineering, St Thomas' Hospital, King's College London, UK

Y. Lecarpentier

Institut du Coeur, Hôpital de la Pitié-Salpêtrière, Paris, and Centre de Recherche Clinique, Hôpital de Meaux, France observed at different scales and at the local vs. organ levels.

Keywords cardiac modeling; experimental validation; hierarchical modeling; length-dependence effects; Frank-Starling mechanism

\section{Introduction}

Complex three-dimensional (3D) multi-physics beating heart models are now available - see, e.g., (Peskin, 1975; Nash and Hunter, 2000; Costa et al, 2001; Sachse, 2004; Kerckhoffs et al, 2005; Sainte-Marie et al, 2006; Niederer and Smith, 2009) and references therein - including for patient-specific simulations as in (Smith and et al., 2011; Chabiniok et al, 2011), themselves based on various inverse modeling approaches, see (Schmid et al, 2008; Moireau et al, 2008). However, such models are computationally intensive, and their physical and computational complexities make their detailed validations and calibrations difficult. Preliminary calibrations of the numerous physical parameters are essential to run meaningful simulations, and to initiate inverse modeling loops for personalization purposes, and it is very ineffective to perform this preliminary stage with the full 3D model.

Moreover, increasingly sophisticated biomechanical cardiac tissue models - frequently based on multi-scale approaches - aim at capturing ever subtler aspects of the cardiac behavior, whether in physiological or pathological conditions, see e.g. recent survey in (Trayanova and Rice, 2011). This holds in particular for load-dependence mechanisms, which have been recognized as crucial in cardiac physiology for decades, most notably with the so-called Frank-Starling effect in contraction (Frank, 1895; Starling, 1918), and also more recently 
in the relaxation stage (Brutsaert et al, 1980). Various models have endeavored to incorporate such effects, see e.g. (Panerai, 1980) and references therein for contraction, and (Izakov et al, 1991; Niederer et al, 2006) for relaxation. Clearly, as regards the detailed validation of such refined models, the complete organ is not the adequate scale for model assessments based on experimental testing, whereas well-adapted controllable protocols are available at a more local scale, namely, with tissue samples (Lecarpentier et al, 1979; Kentish et al, 1986; Parikh et al, 1993), or even myocytes (Bluhm et al, 1995; Cazorla et al, 2000; Iribe et al, 2006). Nevertheless, while very refined models can be formulated and calibrated to reproduce a given family of experiments, it is also essential that the modeling approach be integrated in a unified framework within which the detailed $3 \mathrm{D}$ behavior of the whole organ can also be assessed, in order to investigate the relations between the behaviors at the local and global scales.

In this paper we will demonstrate how, using geometrical arguments, a generic 3D model that contains the most important ingredients to reproduce a prototypical cardiac cycle can be used to derive associated reduced-dimensional models both in "0D" (zero-dimensional) - typically, to represent a cardiac cavity, or several coupled cavities - and in "1D" (one-dimensional) - to model elongated structures such as fibers or myocytes. Such hierarchical models are intended for use in combination with 3D models to provide dramatic effectiveness gains without compromising modeling accuracy at the local scale, and we emphasize that our procedure could easily be applied to many 3D models. Clearly, whereas the 1D model has a claim to accuracy for myocardial structures of adequate geometries, the $0 \mathrm{D}$ model is only meant to provide a straightforward translation of local properties to the organ level without incorporating any anatomical details - whereas other reduced-dimensional approaches such as in (Arts et al, 1991; Lumens et al, 2009) include more detailed anatomical descriptions - while the $3 \mathrm{D}$ model is available for accurate simulations of the whole organ. We emphasize, indeed, that the major originality and potential of our approach lie in that it allows exploiting a combination of several such closely-related models, within the hierarchical family thus-constructed, for different purposes such as:

- 1D-0D: to obtain fast translations of experimentally assessed properties - based on 1D samples - to the "organ" level approximately represented by the 0D model;

- 1D-3D: to infer much more accurate translations to the organ level, e.g. including spatial heterogeneities and detailed fiber distributions;
- 0D-3D: to easily calibrate the constitutive properties based on global indicators prior to running 3D simulations.

We choose to illustrate this dimensional reduction strategy with the 3D model originally proposed in (SainteMarie et al, 2006) and further refined in (Chapelle et al, 2012), and we then endeavor to use the resulting 1D reduced model to assess the modeling framework against unpublished experimental data obtained with rat papillary muscle samples. The experimental protocol is specifically designed to mimic a cardiac cycle under varying preload and afterload conditions, in order to more particularly investigate load-dependence effects. In our model, systolic effects are accounted for by a function - proposed in (Chapelle et al, 2012) - representing the varying number of available cross-bridges with respect to sarcomere strain, which we validate with detailed measured trajectories of extensions and forces, and also with Hill-type force-velocity curves. Concerning the load-dependence effects occurring in diastole, we incorporate into the model a new ingredient inspired from (Izakov et al, 1991).

Once the biophysical model parameters have been calibrated using the 1D model confronted with papillary muscles experimental data, we reemploy these parameters - with some limited adaptations - in the 0D model in order to explore the corresponding behavior of a cardiac cavity representing a left ventricle. In particular, we assess the end-systolic pressure-volume relationship (ESPVR), which our hierarchical modeling strategy allows to directly relate to the length-dependence effects and Frank-Starling mechanism. We also analyse the resulting behavior in the light of the varying elastance concept (Suga et al, 1973).

The paper is organized as follows. In Section 2 we recall the main ingredients of the $3 \mathrm{D}$ cardiac model proposed in (Sainte-Marie et al, 2006; Chapelle et al, 2012). Next, in Section 3 we present the derivation of the reduced-dimensional $0 \mathrm{D}$ and $1 \mathrm{D}$ models from the 3D formulation. Then, in Section 4 (Results) we describe the experimental setup and report on our validation trial based on the 1D model, before assessing the behavior of the $0 \mathrm{D}$ cavity model. These results are further discussed in Section 5, and we finally give some concluding remarks in Section 6.

\section{3D model summary}

We consider the multiscale cardiac model proposed in (Sainte-Marie et al, 2006; Chapelle et al, 2012), of which we now summarize the main ingredients, while 
also further emphasizing the distinctions that we introduce in this work.

\subsection{Sarcomere behavior}

Muscles present a multiscale fiber-based structure. At the microscale, each fiber exhibits a striated aspect resulting from the succession of contractile units called sarcomeres. Within the sarcomeres, myosin molecular motors gathered in so-called thick filaments can periodically attach to the surrounding thinner actin-made filaments - thus creating so-called cross-bridges - in the presence of adenosine tri-phosphate (ATP), the metabolic fuel of cells. The forces exerted via these cross-bridges by actin-myosin interaction can then induce the relative sliding of the interdigitated filaments, hence the shortening of individual sarcomeres and the macroscopic contraction.

We first concentrate on the behavior of the sarcomeres and model the kinetics of cross-bridges by using an extension of A.F. Huxley's model (Huxley, 1957). Let us denote by $e_{c}$ the equivalent strain associated with the relative displacements of actin versus myosin filaments. All along the thick myosin filaments, the protruding myosin heads can attach to special sites located on thin actin filaments. For a given myosin head, we denote by $s$ the distance to the closest such actin site scaled by a characteristic interspace distance. Assuming that only one site at a time is available for any given myosin head, we introduce $n(s, t)$ the fraction of heads attached at a distance $s$ at time $t$. As long as a head remains attached, its extension $s$ varies at the same rate as $e_{c}$, hence,

$\frac{\partial n}{\partial t}+\dot{e}_{c} \frac{\partial n}{\partial s}=\left(n_{0}\left(e_{c}\right)-n\right) f-n g$,

where $f$ and $g$ denote binding and unbinding rates, respectively, and the strain-dependent function $n_{0}$ accounts for the length-dependent fraction of recruitable myosin heads, by which we depart from the original Huxley equation with right-hand side $(1-n) f-n g$. Many earlier works have proposed mechanical models of muscle contraction based on the Huxley description or variants thereof, albeit in general the introduction of such terms as $n_{0}$ in (1) primarily aims at providing a detailed description of calcium dynamics (Wong, 1972; Zahalak and Ma, 1990), in which some length-dependence can be optionally introduced (Panerai, 1980). Here, with our focus on mechanical modeling we will consider chemical activation as given, and directly incorporate length dependence into $n_{0}$. The heart muscle is known to work on the "ascending limb" of the force-length relation where the maximum active force rapidly increases with the degree of myofilament overlap within the sarcomeres (Gordon et al, 1966; Fabiato and Fabiato, 1975; Julian and Sollins, 1975; ter Keurs et al, 1980; Kentish et al, 1986; Shiels and White, 2008). This phenomenon - related to the Frank-Starling mechanism at the organ level - is of utmost importance for the cardiac function (Moss and Fitzsimons, 2002; Guyton and Hall, 2011; Tortora and Derrikson, 2009) and will be characterized by $n_{0}\left(e_{c}\right)$ in our model. Moreover, as in (Bestel et al, 2001; Chapelle et al, 2012) we model $f$ and $g$ by

$$
\left\{\begin{array}{l}
f(s, t)=|u|_{+} \mathbb{1}_{s \in[0,1]} \\
g(s, t)=|u|_{+} \mathbb{1}_{s \notin[0,1]}+|u|_{-}+\alpha\left|\dot{e}_{c}\right|
\end{array}\right.
$$

where $\mathbb{1}$ denotes the indicator function - namely, e.g., $\mathbb{1}_{s \in[0,1]}=1$ for $s \in[0,1]$, and 0 otherwise - and $u$ denotes a variable reaction rate summarizing chemical activation - in particular calcium kinetics, see e.g. (Zahalak and Ma, 1990; Hunter et al, 1998) - inducing contraction or relaxation depending on whether $u$ is positive or negative, respectively. We use $|u|_{+}$et $|u|_{-}$ to respectively denote the positive and absolute values of $u$, namely, $|u|_{+}=u$ when $u \geq 0$ and 0 otherwise, whereas $|u|_{-}=-u$ when $u \leq 0$ and 0 otherwise. The term $\alpha\left|\dot{e}_{c}\right|$ accounts for bridges destruction upon rapid length changes, which is revealed by rapid force drop following fast length change (Izakov et al, 1991).

With this particular choice of $f$ and $g$, we have $f+$ $g=|u|+\alpha\left|\dot{e}_{c}\right|$ and we note that this expression is independent of $s$, an assumption often used in modeling actin-myosin interaction (Guerin et al, 2011).

Relaxation is also known to be load-dependent in mammalian cardiac muscles, with experimental evidence showing that relaxation occurs earlier at lower loads or smaller sarcomere lengths (Lecarpentier et al, 1979). This phenomenon is often attributed to collective effects induced by an interplay between the ability of troponin to bind calcium ions and the concentration of myosin motors, or by steric effects due to changes in lattice spacing upon contraction (Izakov et al, 1991; Campbell, 2011), and also by calcium concentration variations regulated by ion exchangers and sarcoplasmic reticulum (Brutsaert et al, 1980). This additional length-dependence effect motivates that we depart from the previous formulations (Chapelle et al, 2012; SainteMarie et al, 2006) and represent this phenomenon by introducing in the spirit of (Izakov et al, 1991) - albeit in a simpler summarized manner - a new internal variable $w(t)$ obeying the first-order dynamics

$\alpha_{r} \dot{w}=m_{0}\left(e_{c}\right)-w$, 
which has a multiplicative effect when $u \leq 0$ (relaxation), namely,

$u=|\bar{u}(t)|_{+}-w|\bar{u}(t)|_{-}$,

where $\bar{u}(t)$ is now an input variable independent of the sarcomere state. The relaxation load-dependence is then driven by the function $m_{0}$ that will be defined as a decreasing function of $e_{c}$ with a reference value of 1 at high $e_{c}$, while the parameter $\alpha_{r}$ is a time constant associated with a simple delay effect pertaining to the complex chain of underlying chemical processes summarized in the evolution of $w$.

The stress state in the sarcomere is then obtained by assuming here a simple quadratic energy for realized cross-bridges, in the form

$W_{m}(s)=\frac{k_{0}}{2}\left(s+s_{0}\right)^{2}$,

namely, a linear spring of stiffness $k_{0}$ and pre-strain $s_{0}$, which induces an individual force $k_{0}\left(s+s_{0}\right)$. The overall stiffness and stress in the sarcomere are thus respectively given by

$k_{c}(t)=k_{0} \int n(s, t) d s$,

$\tau_{c}(t)=\int W_{m}^{\prime}(s) n(s, t) d s=k_{0} \int\left(s+s_{0}\right) n(s, t) d s$,

where we recognize expressions directly associated with the first two moments of the density function $n$. The corresponding moments dynamics are then obtained by integrating over $s$ in (1), which leads to the following closed-form dynamical system

$\left\{\begin{array}{l}\dot{k}_{c}=-\left(|\bar{u}|_{+}+w|\bar{u}|_{-}+\alpha\left|\dot{e}_{c}\right|\right) k_{c}+n_{0} k_{0}|\bar{u}|_{+} \\ \dot{\tau}_{c}=-\left(|\bar{u}|_{+}+w|\bar{u}|_{-}+\alpha\left|\dot{e}_{c}\right|\right) \tau_{c}+n_{0} \sigma_{0}|\bar{u}|_{+}+k_{c} \dot{e}_{c}\end{array}\right.$

where $\sigma_{0}=k_{0}\left(s_{0}+1 / 2\right)$ represents the maximum active stress.

\subsection{Overall constitutive law}

We denote by $y$ the displacement field with respect to a stress-free reference configuration $\Omega_{0}$. We then introduce the right Cauchy-Green deformation tensor $\underline{\underline{C}}$, and the associated Green-Lagrange strain tensor $\underline{\underline{e}}$, $\overline{\mathrm{de}}-$ fined by

$\underline{\underline{C}}=\underline{\underline{F}}^{T} \cdot \underline{\underline{F}}, \quad \underline{\underline{e}}=\frac{1}{2}(\underline{\underline{C}}-\underline{\underline{I}})$,

where $\underline{F}=\underline{I}+\nabla y$ is the deformation gradient.

As in (Sainte-Marie et al, 2006; Chapelle et al, 2012), we use a non-linear Hill-Maxwell rheological law to incorporate the above contractile modeling into the overall behavior, see Fig.1. As we are pursuing a Lagrangian

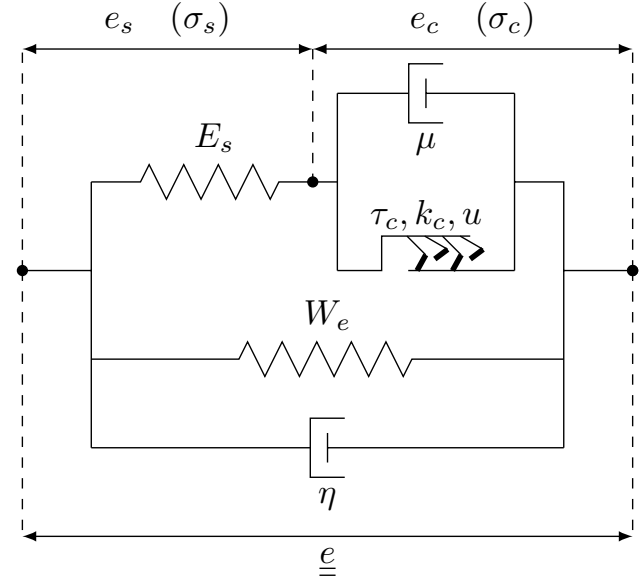

Fig. 1 Hill-Maxwell rheological model

description of the system with a strain measure given by the Green-Lagrange strain tensor, it is natural to use the second Piola-Kirchhoff stress tensor for internal forces, and except when otherwise specified all stress quantities considered will be associated with this specific stress tensor. In the first branch of this rheological schematic, the contractile element is placed in series with a linear elastic element, and in parallel with a linear damping element. This whole branch is assumed to be of $1 \mathrm{D}$ character, namely, producing stresses only along the fiber direction - represented by a spatiallyvarying unit vector $\underline{\tau}_{1}-$ in the form $\sigma_{1 \mathrm{D}} \underline{\tau}_{1} \otimes \underline{\tau}_{1}$, in relation to strains measured along the same direction, i.e. $e_{1 \mathrm{D}}=\underline{\tau}_{1} \cdot \underline{\underline{e}} \cdot \underline{\tau}_{1}$. The parallel association of the viscous component gives

$\sigma_{c}=\tau_{c}+\mu \dot{e}_{c}$

with $\mu$ a viscous damping parameter. Concerning the series linearly-elastic element characterized by the stressstrain law $\sigma_{s}=E_{s} e_{s}$, in our non-linear large strain framework, the $1 \mathrm{D}$ strains $e_{1 \mathrm{D}}, e_{c}$ and $e_{s}$ are related by

$1+2 e_{1 \mathrm{D}}=\left(1+2 e_{s}\right)\left(1+2 e_{c}\right)$,

while the stresses $\sigma_{1 \mathrm{D}}, \sigma_{s}$ and $\sigma_{c}$ obey

$\sigma_{1 \mathrm{D}}=\frac{\sigma_{s}}{1+2 e_{c}}=\frac{\sigma_{c}}{1+2 e_{s}}$,

these relations representing the nonlinear extensions of the usual series-type rheological identities (Chapelle et al, 2012). This leads to the additional dynamical relation

$\tau_{c}+\mu \dot{e}_{c}=E_{s} \frac{\left(e_{1 \mathrm{D}}-e_{c}\right)\left(1+2 e_{1 \mathrm{D}}\right)}{\left(1+2 e_{c}\right)^{3}}$.

To take into account the connective tissue surrounding the myocardial fiber, we introduce a hyperelastic 
potential $W_{e}$ and a viscous pseudopotential $W_{v}$ and define the fully $3 \mathrm{D}$ passive stress as follows

$\underline{\underline{\Sigma}}_{p}=\frac{\partial W_{e}}{\partial \underline{\underline{e}}}(\underline{\underline{e}})+\frac{\partial W_{v}}{\partial \underline{\underline{\dot{e}}}}(\underline{\underline{e}}, \underline{\underline{\dot{e}}})$.

Here, we consider a hyperelastic potential of transverse isotropic type $W_{e}\left(J_{1}, J_{2}, J_{4}\right)$, function of the classical reduced invariants of the Cauchy-Green strain tensor, which are expressed with the standard invariants $I_{1}, I_{2}, I_{3}$ and $I_{4}$ as

$J_{1}=I_{1} I_{3}^{-\frac{1}{3}}, \quad J_{2}=I_{2} I_{3}^{-\frac{1}{3}}, \quad J_{3}=I_{3}^{\frac{1}{2}}, \quad J_{4}=I_{4} I_{3}^{-\frac{1}{3}}$

where

$$
\begin{array}{ll}
I_{1}=\operatorname{tr}(\underline{\underline{C}}), & I_{2}=\frac{1}{2}\left(\operatorname{tr}(\underline{\underline{C}})^{2}-\operatorname{tr}\left(\underline{\underline{C}}^{2}\right)\right), \\
I_{3}=\operatorname{det}(\underline{\underline{C}}), & I_{4}=\underline{\tau}_{1} \cdot \underline{\underline{C}} \cdot \underline{\tau}_{1} .
\end{array}
$$

We assume the material to be incompressible, namely, $I_{3}=J_{3}=1$, which is why $W_{e}$ is taken independent of $J_{3}$. While the terms containing $J_{1}$ and $J_{2}$ are isotropic in nature, the $J_{4}$ invariant accounts for the privileged direction associated with the fiber - namely, transverse isotropic behavior - that can result from the contribution of titin and the intercellular collagen network (Linke et al, 1994; Weiwad et al, 2000; Fukuda et al, 2001; Linke and Fernandez, 2002). In order to derive the passive stress $\underline{\underline{\Sigma}}_{p}$, for the hyperelastic part we apply the chain rule

$\frac{\partial W_{e}}{\partial \underline{\underline{e}}}=2 \sum_{i}\left(\frac{\partial W_{e}}{\partial J_{i}}\right)\left(\frac{\partial J_{i}}{\partial \underline{\underline{C}}}\right)$,

where we use the classical expressions of the invariant derivatives

$$
\left\{\begin{array}{l}
\frac{\partial J_{1}}{\partial \underline{\underline{C}}}=I_{3}^{-\frac{1}{3}}\left(\underline{\underline{I}}-\frac{1}{3} I_{1} \underline{\underline{C}}^{-1}\right) \\
\frac{\partial J_{2}}{\partial \underline{\underline{C}}}=I_{3}^{-\frac{2}{3}}\left(I_{1} \underline{\underline{I}}-\underline{\underline{C}}-\frac{2}{3} I_{2} \underline{\underline{C}}^{-1}\right) \\
\frac{\partial \bar{J}_{4}}{\partial \underline{\underline{C}}}=I_{3}^{-\frac{1}{3}}\left(\underline{i}_{\phi_{1}} \otimes \underline{i}_{\phi_{1}}-\frac{1}{3} I_{4} \underline{\underline{C}}^{-1}\right)
\end{array}\right.
$$

As for the viscous pseudo-potential we will consider the simple form $W_{v}=\frac{\eta}{2} \operatorname{tr}(\underline{\underline{e}})^{2}$. Following the classical approach, in order to handle incompressibility we will introduce a pressure-type Lagrange multiplier $p$ such that the overall second Piola-Kirchhoff stress tensor can be written

$\underline{\underline{\Sigma}}=\underline{\underline{\Sigma}}_{p}+\sigma_{1 \mathrm{D}} \underline{\tau}_{1} \otimes \underline{\tau}_{1}-p \underline{\underline{C}}^{-1}$,

where

$\sigma_{1 \mathrm{D}}=\frac{1+2 e_{c}}{1+2 e_{1 \mathrm{D}}}\left(\tau_{c}+\mu \dot{e}_{c}\right)$.

\subsection{System equilibrium}

The overall mechanical behavior is governed by the fundamental law of dynamics, written in the deformed configuration $\Omega$ as

$\operatorname{div} \underline{\underline{\sigma}}+\rho(\underline{f}-\underline{\gamma})=0$,

where $\underline{\underline{\sigma}}$ is the Cauchy stress tensor, related to $\underline{\underline{\Sigma}}$ by

$\underline{\underline{\sigma}}=J^{-1} \underline{\underline{F}} \cdot \underline{\underline{\Sigma}} \cdot \underline{\underline{F}}^{T}$,

$\rho$ is the mass density of the tissue, $f$ is the external force per unit mass and $\underline{\gamma}$ the acceleration. This should be supplemented with boundary conditions in the form

$\underline{\underline{\sigma}} \cdot \underline{\nu}=\underline{g}$

where $\underline{\nu}$ denotes the outward unit normal vector and $\underline{g}$ prescribed forces on some parts of the boundary, e.g. of pressure type, namely, $g=-P \underline{\nu}$ in this case. The corresponding weak formulation - also called principle of virtual work - can be written in a Lagrangian formalism, namely, in $\mathcal{V}$ the space of admissible displacements measured with respect to the reference configuration $\Omega_{0}$ as

$\mathcal{P}_{a}\left(\underline{y}^{*}\right)+\mathcal{P}_{i}\left(\underline{y}^{*}\right)=\mathcal{P}_{e}\left(\underline{y}^{*}\right), \quad \forall \underline{y}^{*} \in \mathcal{V}$,

with

$$
\begin{aligned}
& \mathcal{P}_{a}\left(\underline{y}^{*}\right)=\int_{\Omega_{0}} \rho \underline{\ddot{y}} \cdot \underline{y}^{*} d \Omega, \\
& \mathcal{P}_{i}\left(\underline{y}^{*}\right)=\int_{\Omega_{0}} \underline{\underline{\Sigma}}: d_{\underline{y}} \underline{\underline{e}} \cdot \underline{y}^{*} d \Omega, \\
& \mathcal{P}_{e}\left(\underline{y}^{*}\right)=-\int_{S_{\text {endo }}} P_{v} \underline{\nu}_{0} \cdot \underline{\underline{F}}^{-1} \cdot \underline{y}^{*} d S,
\end{aligned}
$$

where $y^{*}$ represents a displacement test function, and $P_{v}$ the intraventricular blood pressure exerting forces on the endocardium surfaces $S_{\text {endo }}$ of outward unit normal vector $\underline{\nu}_{0}$. The virtual increment of the nonlinear Green-Lagrange strain tensor $\underline{\underline{e}}$ is given by the differential

$d_{\underline{y} \underline{\underline{e}}} \cdot \underline{y}^{*}=\frac{1}{2}\left(\underline{\underline{F}}^{T} \cdot \nabla \underline{y}^{*}+\left(\nabla \underline{y}^{*}\right)^{T} \cdot \underline{\underline{F}}\right)$.

The internal pressure $P_{v}$ is coupled to the cardiac outflow $Q=-\dot{V}$, where $V$ is the ventricular cavity volume, by additional conditions. Inflows and outflows are made possible by opening and closing the cavity valves, which occurs depending on the balance of internal and external pressures summarized as (Sainte-Marie et al, 2006)

$-\dot{V}=Q=q\left(P_{v}, P_{a r}, P_{a t}\right)$, 
where $P_{a r}$ denotes the pressure in the aorta or in the pulmonary artery, depending on the ventricle considered - i.e., the afterload $-P_{a t}$ is the corresponding atrial pressure that gives the preload in the filling phase, and $q$ is a regularized version - for numerical purposes - of the following ideal behavior

$$
\begin{cases}Q \leq 0 & \text { if } P_{v}=P_{a t} \text { (filling) } \\ Q=0 & \text { if } P_{a t} \leq P_{v} \leq P_{a r} \text { (isovol. phases) } \\ Q \geq 0 & \text { if } P_{v} \geq P_{a r} \text { (ejection) }\end{cases}
$$

that we approximate as

$$
\left\{\begin{array}{l}
Q=K_{a t}\left(P_{v}-P_{a t}\right), \quad \text { if } P_{v} \leq P_{a t} \\
Q=K_{p}\left(P_{v}-P_{a t}\right), \quad \text { if } P_{a t} \leq P_{v} \leq P_{a r} \\
Q=K_{a r}\left(P_{v}-P_{a r}\right)+K_{p}\left(P_{a r}-P_{a t}\right), \quad \text { if } P_{v} \geq P_{a r}
\end{array}\right.
$$

where the regularizing constants $K_{a t}, K_{p}$ and $K_{a r}$ must be chosen such that $K_{p}$ is much smaller than $K_{a r}$ and $K_{a t}$ to ensure that the flow is negligible in isovolumic phases.

Finally, the system is closed by a relation representing the external circulation, with a so-called two-stage Windkessel model written as

$$
\left\{\begin{array}{l}
C_{p} \dot{P}_{a r}+\left(P_{a r}-P_{d}\right) / R_{p}=Q \\
C_{d} \dot{P}_{d}+\left(P_{d}-P_{a r}\right) / R_{p}=\left(P_{v s}-P_{d}\right) / R_{d}
\end{array}\right.
$$

where $C_{p}, R_{p}, C_{d}$ and $R_{d}$ denote capacitances and resistances of the proximal and distal circulations, $P_{d}$ denotes an additional pressure variable called distal pressure, and $P_{v s}$ is a constant representing the venous system pressure.

We can now summarize all the above 3D modeling equations in the following system

$$
\left\{\begin{array}{l}
\mathcal{P}_{a}\left(\underline{y}^{*}\right)+\mathcal{P}_{i}\left(\underline{y}^{*}\right)=\mathcal{P}_{e}\left(\underline{y}^{*}\right), \quad \forall \underline{y}^{*} \in \mathcal{V} \\
\underline{\underline{\Sigma}}=\frac{\partial W_{e}}{\partial \underline{\underline{e}}}+\frac{\partial W_{v}}{\partial \underline{\underline{\dot{e}}}}+\sigma_{1 \mathrm{D}} \underline{\tau}_{1} \otimes \underline{\tau}_{1}-p \underline{\underline{C}} \\
\sigma_{1 \mathrm{D}}=E_{s} \frac{e_{1 \mathrm{D}}-e_{c}}{\left(1+2 e_{c}\right)^{2}} \\
\left(\tau_{c}+\mu \dot{e}_{c}\right)=E_{s} \frac{\left(e_{1 \mathrm{D}}-e_{c}\right)\left(1+2 e_{1 \mathrm{D}}\right)}{\left(1+2 e_{c}\right)^{3}} \\
\dot{k}_{c}=-\left(|\bar{u}|_{+}+w|\bar{u}|_{-}+\alpha\left|\dot{e}_{c}\right|\right) k_{c}+n_{0} k_{0}|\bar{u}|_{+} \\
\dot{\tau}_{c}=-\left(|\bar{u}|_{+}+w|\bar{u}|_{-}+\alpha\left|\dot{e}_{c}\right|\right) \tau_{c}+n_{0} \sigma_{0}|\bar{u}|_{+}+k_{c} \dot{e}_{c} \\
-\dot{V}=Q=q\left(P_{v}, P_{a r}, P_{a t}\right) \\
C_{p} \dot{P}_{a r}+\left(P_{a r}-P_{d}\right) / R_{p}=Q \\
C_{d} \dot{P}_{d}+\left(P_{d}-P_{a r}\right) / R_{p}=\left(P_{v s}-P_{d}\right) / R_{d}
\end{array}\right.
$$

\section{Reduced formulations}

Dimensional reduction is a process by which the dimension of the spatial variables space in which the model is posed - 3D in our case - is decreased by making adequate assumptions, kinematical and otherwise, concerning the dimensions that are "eliminated" in the reduction process. A prototypical example of this is provided by structural modeling in mechanics, see e.g. (Bathe, 1996; Chapelle and Bathe, 2011) and references therein. As is well-known in structural mechanics, indeed, dramatic gains in computational effectiveness can thus be obtained, together with very limited loss in accuracy provided the underlying assumptions are adequately justified. The dimensional reduction process, however, is also known to be quite intricate when nonlinear constitutive equations are considered, a difficulty that we must address here. In our case we will demonstrate two such possible reductions:

- 0D model: assuming spherical symmetry we will obtain a 0D model, namely, without any spatial variable; this model aims at approximately representing a cardiac cavity - the left ventricle, typically - but of course as spherical symmetry does not hold in actuality, the model will have limited accuracy, and in fact is only meant as a fast simulation tool exhibiting adequate trends in behavior, in particular as regards parameter variations.

- 1D model: assuming cylindrical symmetry and uniaxial loading we will derive a $1 \mathrm{D}$ model; in this case the reduced model can be very accurate in specific contexts where the assumptions are justified, such as experimental testing with ad hoc muscle samples, as will be considered in Section 4 .

We point out that in the latter case such a $1 \mathrm{D}$ reduced model is the best-suited candidate for confronting muscle model simulations to experimental measurements obtained with cylindrical samples. In fact, a "naive" use of the 3D model instead - with one finite element across the thickness, say - would lead to severely erroneous modeling results due to fundamental incompatibilities between simplified kinematics and the actual stress state, see (Koiter, 1965; Chapelle and Bathe, 2011). Therefore, in such cases strong 3D mesh refinement would be required, whereas just a few 1D elements - or even a single element when a homogeneous behavior is considered - can suffice to provide excellent accuracy.

We further emphasize that our reduction strategy is not a simple application of local 3D constitutive equations in specific configurations, but incorporates essential modeling ingredients into complete formulations 
which retain the character of continuous media dynamics, compatible in particular with relevant boundary conditions, and with incompressibility constraints. Moreover this approach is generic, and can be applied with a wide class of models, indeed.

\subsection{D-formulation}

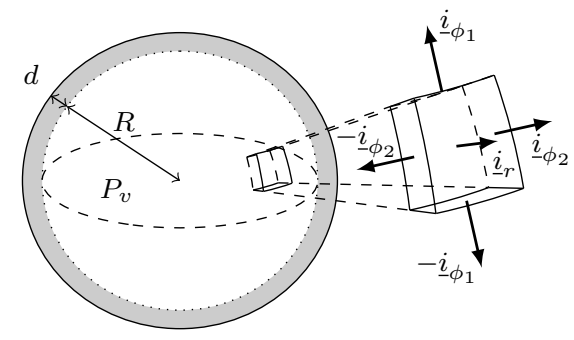

Fig. 2 Spherical model of a ventricle

\subsubsection{Geometry and kinematics}

We define a simplified problem in which the geometry and the physical behavior are endowed with spherical symmetry properties. The simplified geometry for a ventricle is given by a sphere of radius $R_{0}$ and thickness $d_{0}$ in the stress-free reference configuration $\Omega_{0}$, see Fig.2. Assuming that the constitutive properties also exhibit spherical symmetry, the resulting behavior under an internal pressure loading will display the same symmetry, and the deformed configuration is then characterized by modified radius $R$ and thickness $d$. At any given point in $\Omega_{0}$, we denote by $\left(\underline{i}_{r}, \underline{i}_{\phi_{1}}, \underline{i}_{\phi_{2}}\right)$ an orthonormal basis, with $\underline{i}_{r}$ radial and $\left(\underline{i}_{\phi_{1}}, \underline{i}_{\phi_{2}}\right)$ orthoradial defined so that $\underline{i}_{\phi_{1}}=\underline{\tau}_{1}$, i.e. the fiber direction. With the above assumptions the displacement field with respect to the reference configuration is radial, namely, given by $\underline{y}=y \underline{i}_{r}=\left(R-R_{0}\right) \underline{i}_{r}$, and the right CauchyGreen deformation tensor has the special form

$\underline{\underline{C}}=\left(\begin{array}{ccc}C_{r r} & 0 & 0 \\ 0 & C & 0 \\ 0 & 0 & C\end{array}\right)$,

where $\sqrt{C}=1+y / R_{0}$ is the ratio of circumferential lengths between the reference and deformed configurations. Assuming incompressible behavior, we have $\operatorname{det} \underline{C}=1$, hence $C_{r r}=C^{-2}$. Considering a virtual displacement $\underline{y}^{*}=y^{*} \underline{i}_{r}$ with the above-assumed kinematic symmetry, we find in each orthoradial direction

$\left(d_{\underline{y}} \underline{e} \cdot \underline{y}^{*}\right)_{\phi \phi}=\left(1+y / R_{0}\right)\left(y^{*} / R_{0}\right)$.

\subsubsection{Stress and equilibrium derivation}

Due to the relative thin-ness of the wall compared to the sphere radius, classical arguments of shell theory justify that the radial stress $\Sigma_{r r}$ can be neglected compared to the orthoradial components (Chapelle and Bathe, 2011). Therefore, in the decomposition (6) the Lagrange multiplier $p$ can be explicitly inferred from $\Sigma_{r r}=0$, viz.

$p=C^{-2}(\underline{\underline{\Sigma}} p)_{r r}$.

Hence, in (13a) the power of internal forces, $\mathcal{P}_{i}$ per unit volume gives, by using (15),

$$
\begin{aligned}
\underline{\underline{\Sigma}}: d_{\underline{y}} \underline{\underline{e}} \cdot \underline{y}^{*} & =\left(1+\frac{y}{R_{0}}\right) \frac{y^{*}}{R_{0}}\left(\Sigma_{\phi_{1} \phi_{1}}+\Sigma_{\phi_{2} \phi_{2}}\right) \\
& =\left(1+\frac{y}{R_{0}}\right) \frac{y^{*}}{R_{0}} \Sigma_{\mathrm{sph}},
\end{aligned}
$$

with the combined stress quantity $\Sigma_{\mathrm{sph}}$ defined by

$$
\Sigma_{\mathrm{sph}}=\left(\underline{\underline{\Sigma}}_{p}\right)_{\phi_{1} \phi_{1}}+\left(\underline{\underline{\Sigma}}_{p}\right)_{\phi_{2} \phi_{2}}+\sigma_{1 \mathrm{D}}-2 C^{-3}\left(\underline{\underline{\Sigma}}_{p}\right)_{r r} .
$$

Note that no radial term appears in the expression of the tensor product $\underline{\underline{\Sigma}}: d_{\underline{y}} \underline{\underline{e}} \cdot \underline{y}^{*}$ due to vanishing radial stresses. The passive stress $\underline{\underline{\Sigma}}$ is directly inferred from the specific choice of the energy functionals $W_{e}$ and $W_{v}$, see (7), and - with the kinematical assumptions made - the invariants and their derivatives reduce to

$$
\begin{aligned}
& \left\{\begin{array}{l}
J_{1}=2 C+C^{-2} \\
J_{2}=C^{2}+2 C^{-1} \\
J_{4}=C
\end{array}\right. \\
& \left\{\begin{array}{l}
\frac{\partial J_{1}}{\partial \underline{\underline{\underline{C}}}}=\underline{\underline{I}}-\frac{1}{3}\left(2 C+C^{-2}\right) \underline{\underline{C}} \\
\frac{\partial \bar{J}_{2}}{\partial \underline{\underline{C}}}=\left(2 C+C^{-2}\right) \underline{\underline{I}}-\underline{\underline{C}}-\frac{2}{3}\left(C^{2}+2 C^{-1}\right) \underline{C^{-1}} \\
\frac{\partial \bar{J}_{4}}{\partial \underline{\underline{C}}}=\underline{i}_{\phi_{1}} \otimes \underline{i}_{\phi_{1}}-\frac{1}{3} C \underline{\underline{C}}^{-1}
\end{array}\right.
\end{aligned}
$$

while the viscous contribution gives

$\frac{\partial W_{v}}{\partial(\underline{\underline{\dot{e}}})}=\frac{\eta}{2} \dot{\underline{C}}$

Substituting into (18) we obtain after simplifications

$$
\begin{aligned}
\Sigma_{\mathrm{sph}}=\sigma_{1 \mathrm{D}}+4\left(1-C^{-3}\right) & \left(\frac{\partial W_{e}}{\partial J_{1}}+C \frac{\partial W_{e}}{\partial J_{2}}\right) \\
+ & 2 \frac{\partial W_{e}}{\partial J_{4}}+\eta \dot{C}\left(1+2 C^{-6}\right),
\end{aligned}
$$

where $\sigma_{1 \mathrm{D}}$ is given by (7). The other terms in (13a) can be integrated directly, hence, we obtain the following 
ordinary-differential equation (ODE) for the displacement $y$

$\rho d_{0} \ddot{y}+\frac{d_{0}}{R_{0}}\left(1+\frac{y}{R_{0}}\right) \Sigma_{\mathrm{sph}}=P_{v}\left(1+\frac{y}{R_{0}}\right)^{2}$.

Remark 1 Our spherical symmetry assumption implicitly requires an isotropic distribution of the fibers in the orthoradial directions. Note that there is no contradiction here with the fact that each point can be associated with a specific fiber direction that corresponds to nonisotropic mechanical behavior at that particular point. This simply means that the local distribution of fibers around that point - e.g. from a probabilistic point of view - should have no privileged direction within the orthoradial plane. This is also justified when assuming that the fiber distribution across the (small) thickness evenly spans all the directions of this plane.

Finally, the valve law (13g) can be expressed as

$-\dot{V}=4 \pi R_{0}^{2}\left(1+\frac{y}{R_{0}}\right)^{2} \dot{y}=f\left(P_{v}, P_{a r}, P_{a t}\right)$,

so that the initial system (13) finally leads to

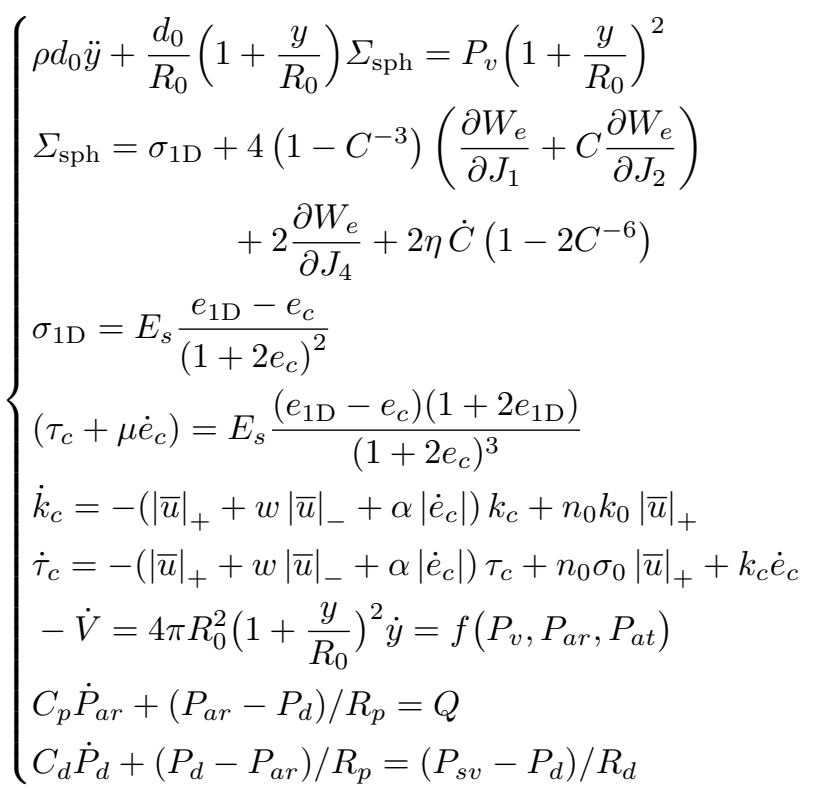

Note that more realistic $0 \mathrm{D}$ models could be derived following similar strategies albeit with more complex geometric descriptors and kinematical assumptions, see e.g. Lumens et al (2009), possibly leading to larger systems of ODEs.

\subsection{D-formulation}

\subsubsection{Geometry and kinematics}

This one-dimensional model aims at reproducing the behavior of an elongated structure made of my-

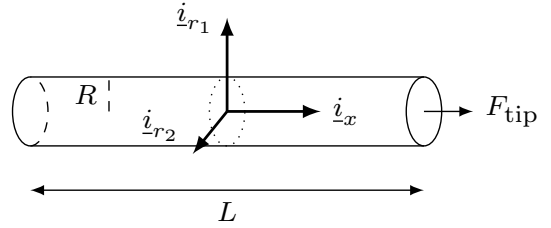

Fig. 3 Cylindrical model of a cardiac muscle sample

ocardium, such as isolated muscle fibers, or even single myocytes, under uniaxial traction. As a simplified geometry we consider a circular cylinder of radius $R_{0}$ and length $L_{0}$ in the reference configuration $\Omega_{0}$, with corresponding values $R$ and $L$ in the deformed configuration, see Fig.3, and we assume that material properties are accordingly endowed with cylindrical symmetry - namely, transverse isotropy - hence, the whole behavior has this same symmetry. As an orthonormal basis we use a first vector $\underline{i}_{x}$ oriented along the fiber - i.e. $\underline{\tau}_{1}=\underline{i}_{x}$ - and we define two arbitrary equivalent directions $\left(\underline{i}_{r_{1}}, \underline{i}_{r_{2}}\right)$ in the cross-section. An external force $F_{\text {tip }}$ is applied at the end of the sample along the $\underline{i}_{x}$-direction, and we seek the resulting longitudinal displacement $y(x)$ at each point of the sample. Due to the incompressibility condition, the Cauchy-Green tensor takes the special form

$\underline{\underline{C}}=\left(\begin{array}{ccc}C & 0 & 0 \\ 0 & C^{-\frac{1}{2}} & 0 \\ 0 & 0 & C^{-\frac{1}{2}}\end{array}\right)$,

where $\sqrt{C}=1+y^{\prime}(x)$ is now the length ratio in the $\underline{i}_{x}$-direction. Therefore in the longitudinal direction we have

$\left(d_{\underline{y}} \underline{\underline{e}} \cdot \underline{y^{*}}\right)_{x x}=\left(1+y^{\prime}\right)\left(y^{*}\right)^{\prime}$,

for a virtual displacement field $\underline{y}^{*}(x)=y^{*}(x) \underline{i}_{x}$.

\subsubsection{Stress and equilibrium derivation}

Considering the cylindrical symmetry and the uniaxial loading condition, it is easily seen that the radial stresses $\Sigma_{r r}$ identically vanish in this case. Like in the 0D model reduction, this allows to compute the Lagrange multiplier $p$, viz.

$p=C^{-\frac{1}{2}}\left(\underline{\underline{\Sigma}}_{p}\right)_{r r}$.

The power of internal forces then reduces to

$\underline{\underline{\Sigma}}: d_{\underline{y}} \underline{\underline{e}} \cdot \underline{y}^{*}=\Sigma_{x x}\left(1+y^{\prime}\right)\left(y^{*}\right)^{\prime}$,

with the axial stress given by

$\Sigma_{x x}=\sigma_{1 \mathrm{D}}+\left(\underline{\underline{\Sigma}}_{p}\right)_{x x}-C^{-\frac{3}{2}}\left(\underline{\underline{\underline{\Sigma}}}_{p}\right)_{r r}$, 
with $e=e_{1 D}=(C-1) / 2$. In this case, we have for the hyperelastic part

$$
\left\{\begin{array}{l}
J_{1}=C+2 C^{-\frac{1}{2}} \\
J_{2}=2 C^{\frac{1}{2}}+C^{-1} \\
J_{4}=C
\end{array}\right.
$$

and

$$
\left\{\begin{array}{l}
\frac{\partial J_{1}}{\partial \underline{\underline{C}}}=1-\frac{1}{3}\left(C+2 C^{-\frac{1}{2}}\right) \underline{\underline{C}}^{-1} \\
\frac{\partial J_{2}}{\partial \underline{\underline{C}}}=\left(C+2 C^{-\frac{1}{2}}\right) \underline{\underline{I}}-\underline{\underline{C}}-\frac{2}{3}\left(2 C^{\frac{1}{2}}+C^{-1}\right) \underline{\underline{C}}^{-1} \\
\frac{\partial \bar{J}_{4}}{\partial \underline{\underline{C}}}=\underline{i}_{x} \otimes \underline{i}_{x}-\frac{1}{3} C \underline{\underline{C}}^{-1}
\end{array}\right.
$$

The derivative of the viscous pseudo-potential gives $\frac{\partial W_{v}}{\partial \underline{\underline{\dot{e}}}}=\frac{\eta}{2} \underline{\underline{\underline{C}}}$.

Then we can rewrite $(21)$ as

$$
\begin{aligned}
\Sigma_{x x}=\sigma_{1 \mathrm{D}}+2\left(1-C^{-\frac{3}{2}}\right) & \left(\frac{\partial W_{e}}{\partial J_{1}}+C^{-\frac{1}{2}} \frac{\partial W_{e}}{\partial J_{2}}\right) \\
+ & 2 \frac{\partial W_{e}}{\partial J_{4}}+\frac{\eta}{2} \dot{C}\left(1+\frac{1}{2} C^{-\frac{9}{4}}\right),
\end{aligned}
$$

and finally we can integrate over each cross-section in (13a), which yields

$$
\int_{0}^{L_{0}}\left[\rho \ddot{y} y^{*}+\Sigma_{x x}\left(1+y^{\prime}\right)\left(y^{*}\right)^{\prime}\right] d x=\frac{F_{\mathrm{tip}}}{A_{0}} y^{*}\left(L_{0}\right),
$$

with $A_{0}=\pi R_{0}^{2}$. As $F_{\text {tip }}$ is assumed to be prescribed here, we are eventually left with the following system

$$
\left\{\begin{array}{l}
\int_{0}^{L_{0}}\left[\rho \ddot{y} y^{*}+\Sigma_{x x}\left(1+y^{\prime}\right)\left(y^{*}\right)^{\prime}\right] d x=\frac{F_{\mathrm{tip}}}{A_{0}} y^{*}\left(L_{0}\right), \forall y^{*} \in \mathcal{V} \\
\Sigma_{x x}=\sigma_{1 \mathrm{D}}+2\left(1-C^{-\frac{3}{2}}\right)\left(\frac{\partial W_{e}}{\partial J_{1}}+C^{-\frac{1}{2}} \frac{\partial W_{e}}{\partial J_{2}}\right) \\
\quad+2 \frac{\partial W_{e}}{\partial J_{4}}+\frac{\eta}{2} \dot{C}\left(1+C^{-\frac{9}{4}}\right) \\
\sigma_{1 \mathrm{D}}=E_{s} \frac{e_{1 \mathrm{D}}-e_{c}}{\left(1+2 e_{c}\right)^{2}} \\
\left(\tau_{c}+\mu \dot{e}_{c}\right)=E_{s} \frac{\left(e_{1 \mathrm{D}}-e_{c}\right)\left(1+2 e_{1 \mathrm{D}}\right)}{\left(1+2 e_{c}\right)^{3}} \\
\dot{k}_{c}=-\left(|\bar{u}|_{+}+w|\bar{u}|_{-}+\alpha\left|\dot{e}_{c}\right|\right) k_{c}+n_{0} k_{0}|\bar{u}|_{+} \\
\dot{\tau}_{c}=-\left(|\bar{u}|_{+}+w|\bar{u}|_{-}+\alpha\left|\dot{e}_{c}\right|\right) \tau_{c}+n_{0} \sigma_{0}|\bar{u}|_{+}+k_{c} \dot{e}_{c}
\end{array}\right.
$$

Note that - when integrating by parts in the variational formulation - we can derive the equivalent strong form of the mechanical equilibrium, namely,

$$
\left\{\begin{array}{l}
\rho \ddot{y}-\left[\Sigma_{x x}\left(1+y^{\prime}\right)\right]^{\prime}=0 \\
\Sigma_{x x}\left(L_{0}\right)\left(1+y^{\prime}\left(L_{0}\right)\right)=\frac{F_{\mathrm{tip}}}{A_{0}}
\end{array}\right.
$$

which represents the counterpart of Eqs. (8a)-(8b).

Remark 2 Cylindrical symmetry is a rather strong assumption, but if we only assume axisymmetry together with "small thickness", classical structural mechanics arguments also lead to vanishing radial stresses, hence a very similar derivation can be performed and the resulting 1D weak form simply reads

$\int_{0}^{L_{0}} A(x)\left[\rho \ddot{y} y^{*}+\Sigma_{x x}\left(1+y^{\prime}\right)\left(y^{*}\right)^{\prime}\right] d x=F_{\mathrm{tip}} y^{*}\left(L_{0}\right)$,

with a non-constant cross-section area parameter $A(x)$.

\section{Results}

\subsection{Application of the 1D model}

We illustrate the use of the above-derived 1D model to represent the behavior of an isolated papillary muscle sample in experimental conditions designed to mimic a cardiac cycle (Sonnenblick, 1962; Lecarpentier et al, 1979). This experimental assessment will concurrently serve the purposes of parameter calibration and model validation, including for fine features such as lengthdependent effects. We will then demonstrate how the corresponding calibration can be used in combination with our dimensional reduction strategy to set up a 0D model of cardiac contraction.

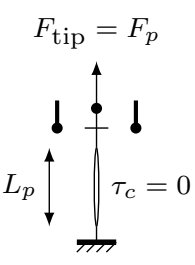

(a) passive

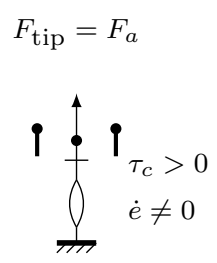

(c) isotonic

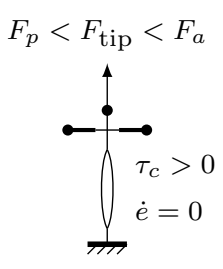

(b) isometric

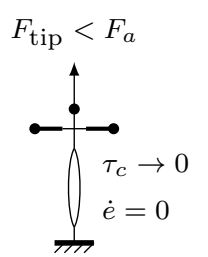

(d) isometric
Fig. 4 Experimental protocol for papillary muscle. (a) passive stretching by preload $F_{p}$; (b) isometric contraction; (c) isotonic contraction and relaxation against prescribed afterload $F_{a} ;(d)$ isometric relaxation

\subsubsection{Experimental data}

We start by briefly describing our experimental protocol before considering the calibration of our 1D-model, see (Claes and Brutsaert, 1971; Brutsaert and Claes, 
1974; De Clerck et al, 1977; Lecarpentier et al, 1979) for a more detailed description. Experiments were performed on a papillary muscle dissected from the left ventricle of an adult rat. The sample length at which the maximum active force is generated $\left(L_{\max }\right)$ is approximately $5 \mathrm{~mm}$, and the cross-section is about $1 \mathrm{~mm}^{2}$. As depicted in Fig.4, the sample is mounted vertically in a chamber and attached at one end, while the other end is connected to an electronically controlled traction device (Brutsaert and Claes, 1974). The displacement of the tip is recorded by a photodiode detector. The chamber is filled with Krebs-Ringer's solution maintained at a temperature of $29^{\circ} \mathrm{C}$. The muscle is stimulated via two platinum electrodes arranged longitudinally around the sample, using rectangular pulses at the rate of $12 \mathrm{~min}^{-1}$, each pulse lasting $5 \mathrm{~ms}$.

In the experimental protocol, the sample in passive state is first stretched to a length $L_{p}$ associated with a preload $F_{p}$, see Fig.4 (a). The muscle is then electrically activated by the above-described pulse. The initial extension, however, is maintained by the control device as long as the measured tip force $F_{\text {tip }}$ is less than a prescribed afterload value $F_{a}$, i.e. this corresponds to isometric contraction, see Fig.4 (b). When this force threshold $F_{a}$ is reached, the muscle is allowed to shorten at fixed tension $F_{\text {tip }}=F_{a}$, namely, in isotonic condition, see Fig.4 (c). Isotonic condition still holds when relaxation occurs, until the sample recovers its initial extension, after which stage further displacements are again prevented, namely, in isometric relaxation, see Fig.4 (d). Note that this sequence is partly non-physiological, as relaxation starts with an isotonic phase, instead of isovolumic in the cardiac cycle.

Two data sets corresponding to two different preloads are available. The largest preload is chosen in order to induce the highest possible active force in the muscle, i.e. $L_{p}$ then corresponds to the so-called $L_{\max }$ for which the length-dependence effect is maximum. For each given preload, the experiment is repeated for several increasing values of the afterload, until fully isometric conditions prevail along the whole cycle. In each such experiment, position and force at the tip are recorded, providing data as shown in Fig.5 (b)-(c).

\subsubsection{Calibration strategy}

We here discuss how we can perform numerical simulations of the 1D-model, and how the model parameters can be calibrated in a systematic manner based on the experimental data.

Discretization procedures. The 1D model proposed in Section 3.2 is discretized time- and space-wise for numerical simulations. Time discretization is performed

\begin{tabular}{llll}
\hline \multicolumn{4}{c}{ 1D-model } \\
\hline$C_{0}, C_{2}$ & $1.910^{3}$ & $C_{1}, C_{3}$ & $1.110^{-1}$ \\
$\sigma_{0}$ & $1.2410^{4}$ & $k_{0}$ & $10^{4}$ \\
$\mu, \eta$ & 70 & $E_{s}$ & $3.10^{7}$ \\
$\alpha$ & 1.5 & $\alpha_{r}$ & 0.12 \\
$A_{0}$ & $10^{-6}$ & $\rho$ & $10^{3}$ \\
\hline 0 D-model (when different from $1 \mathrm{D}-$ model) \\
\hline$R_{0}$ & $2.3610^{-2}$ & $d_{0}$ & $1.4210^{-2}$ \\
$\sigma_{0}$ & $1.2410^{5}$ & $k_{0}$ & $10^{5}$ \\
$R_{p}$ & $8.10^{6}$ & $C_{p}$ & $2.510^{-9}$ \\
$R_{d}$ & $10^{8}$ & $C_{d}$ & $1.10^{-8}$ \\
$K_{a t}$ & $9.10^{-6}$ & $K_{p}$ & $5.10^{-10}$ \\
$K_{a r}$ & $1.310^{-5}$ & $P_{s v}$ & $10^{3}$ \\
\hline
\end{tabular}

Table 1 Calibrated model parameters (in SI units)

using a mid-point time scheme for the displacements and velocities $y$ and $\dot{y}$, and the specific energy-preserving time scheme proposed in (Chapelle et al, 2012) for the internal variables $\left(e_{c}, k_{c}, \tau_{c}\right)$. For spatial discretization we use $P_{1}$-Lagrange finite elements for the displacements, and $\left(e_{c}, k_{c}, \tau_{c}\right)$ are computed at the numerical quadrature points, and condensed out of the linear system being solved at each Newton iteration - within each time step (Sainte-Marie et al, 2006). Assuming that the state of the fiber is approximately homogeneous along the length, we will use a single linear finite element in the simulations. Therefore, the strains and passive stresses will be independent of the position $x$, and so will be the active stress since we only use spacehomogeneous activation patterns in adequacy with the experimental protocol.

Reference configuration. In order to compute deformation indices as used in the model constitutive equation, we need to define a stress-free reference configuration for the sample. This is very delicate due to the extreme compliance of the passive behavior around the reference configuration, and in general this configuration is not properly characterized in such conditions, hence experimental deformations are usually represented with respect to $L_{\max }$ rather than the reference length $L_{0}$. We determined that a reference length set to $L_{0}=0.55 L_{\max }$ allows for consistent adjustment of the passive law based on initial extensions induced by the two preloads. This corresponds to a total GreenLagrange strain at $L_{\max }$ of $e \approx 1.1$, a rather large value. At the sarcomere level, indeed, it is known that the reference length - the so-called slack length - is about $\sim 1.85 \mu \mathrm{m}$, whereas the maximum active stress is measured at sarcomere length $\sim 2.3 \mu \mathrm{m}$, i.e. a strain at $L_{\max }$ 
of $e \approx 0.3$. This discrepancy may be explained by the presence of a compliant series element due to damaged tissue near the clamps, as already reported in (Krueger and Pollack, 1975; ter Keurs et al, 1980). In order to discriminate the effect of such a component, hence to identify its constitutive behavior, we would need simultaneous measurements of sarcomere extensions, which is out of the scope of the present study. Therefore we will pursue our calibration with the above-presented model alone.

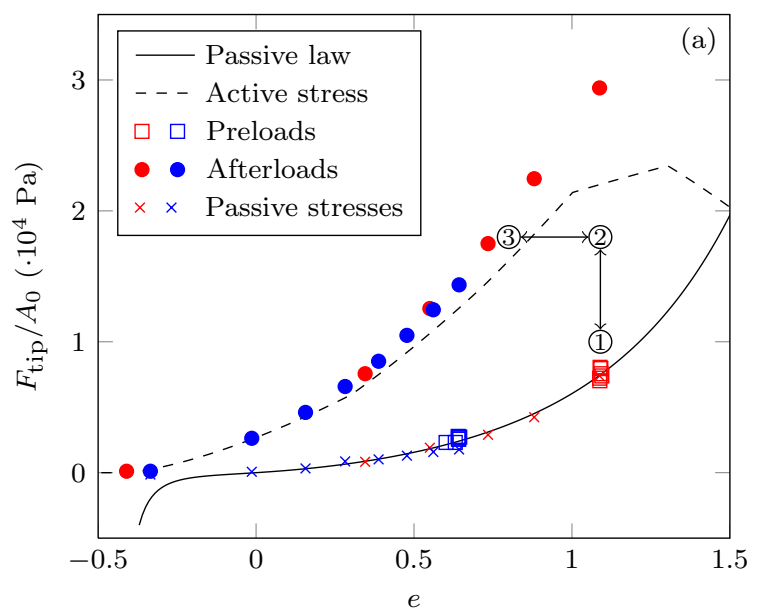

(b)

(c)
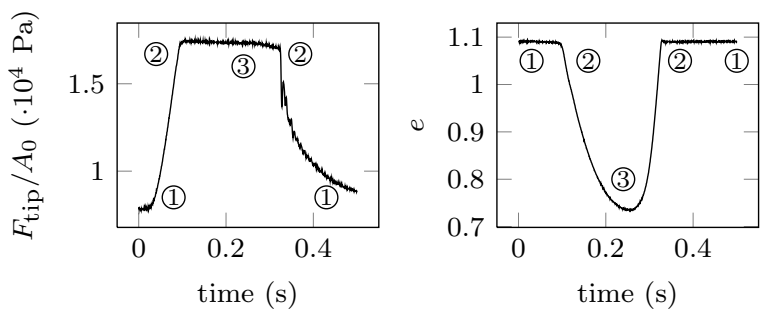

Fig. 5 Calibration of passive and active behavior based on passive extension and maximum active shortening data (a) for high/intermediate values of the preload in red/blue, extracted from typical dynamical measurements for tip forces (b) and strains (c); note: example measurement points marked in (b)(c) are reproduced in (a)

Calibration of the passive behavior law. We calibrate the passive behavior based on the maximum extension points shown in Fig.5 (a) for the two different preload levels (see blue and red squares). The hyperelastic potential $W_{e}$ is taken in the form

$W_{e}=C_{0} \exp \left(C_{1}\left(J_{1}-3\right)^{2}\right)+C_{2} \exp \left(C_{3}\left(J_{4}-1\right)^{2}\right)$,

inspired from (Holzapfel and Ogden, 2009), and the parameters $C_{0}, C_{1}, C_{2}$ and $C_{3}$ are chosen to match the experimental control points, see solid line in Fig.5 (a). We gather the calibrated parameters in Tab.1. Note that with the above type of hyperelastic potential expression, it is not possible to discriminate the parameter values from the two separate exponential terms based on uniaxial type measurements alone. Therefore, we perform this calibration while enforcing the constraint $C_{0}=C_{2}$ and $C_{1}=C_{3}$.

Calibration of the length-dependence effect. The next step consists in the calibration of the length-dependence effect, represented by the function $n_{0}\left(e_{c}\right)$. In Fig.5 (a), the filled circles represent the state of the system at maximum shortening under isotonic conditions, namely, the points labeled " 3 " in the sub-figures (b) and (c). In the context of the heart function, this relation is termed the End Systolic Pressure-Volume Relation (ESPVR). Here, we denote by $e^{\mathrm{sys}}$ and $e_{c}^{\mathrm{sys}}$ the corresponding "end systolic" values of $e$ and $e_{c}$, respectively. This state is characterized by the mechanical equilibrium between the active stress, the passive stress and the afterload $F_{a}$ expressed by combining (21) and (22b), which yields

$\sigma_{1 \mathrm{D}}=\frac{F_{\mathrm{tip}}}{C^{\frac{1}{2}} A_{0}}-\Sigma_{p}, \quad \Sigma_{p}=\left(\underline{\underline{\Sigma}}_{p}\right)_{x x}+C^{-\frac{3}{2}}\left(\underline{\underline{\Sigma}}_{p}\right)_{r r}$,

where $\Sigma_{p}$ denotes the passive part of the second PiolaKirchhoff stress - here 1D, recall (21) - which can be directly inferred from the strain $e^{\text {sys }}$. Hence, (23) gives $\sigma_{1 \mathrm{D}}$ for each "end-systolic" point. Since $\sigma_{1 \mathrm{D}}$ also satisfies $(13 \mathrm{c})$, we can compute the internal strain $e_{c}^{\mathrm{sys}}$. In the mechanical equilibrium considered, $\tau_{c}+\mu \dot{e}_{c}=n_{0}\left(e_{c}\right) \sigma_{0}$, hence Eq.(7) can be rewritten as

$n_{0}\left(e_{c}^{\mathrm{sys}}\right) \sigma_{0}=\sigma_{1 \mathrm{D}} \frac{1+2 e^{\mathrm{sys}}}{1+2 e_{c}^{\mathrm{sys}}}$,

which allows to compute the quantity $n_{0}\left(e_{c}^{\text {sys }}\right) \sigma_{0}$ for any given afterload of associated total strain $e^{\text {sys }}$. The constant $\sigma_{0}$ is inferred from the experimental data at large preload when the device adjusted so that the entire process is isometric (i.e. afterload as large as needed), and using the modeling assumption that $n_{0}\left(e_{c}\right)=1$, namely, the maximum value for this state. Finally, the function $n_{0}$ is then calibrated from all other experimental points using a piecewise linear form, see Fig. 6 where we display this function calibrated for several choices of the series elastic modulus $E_{s}$.

Influence of finite $E_{s}$ coefficient. The effect of having a "finite" value for the series elasticity modulus $E_{s}-$ namely, a value comparable to $\sigma_{0}-$ is that the total strain $e^{\text {sys }}$ differs from the strain of the active component $e_{c}^{\text {sys }}$. In Fig.6, we illustrate this effect by showing the calibrated length-dependence function $n_{0}\left(e_{c}^{\mathrm{sys}}\right)$ and the relation $e^{\text {sys }}$ vs. $e_{c}^{\text {sys }}$ for different values of $E_{s}$, while keeping the same definition of the reference configuration. We can see that the ascending part of the function $n_{0}\left(e_{c}^{\mathrm{sys}}\right)$ becomes steeper as $E_{s}$ decreases, which implies 

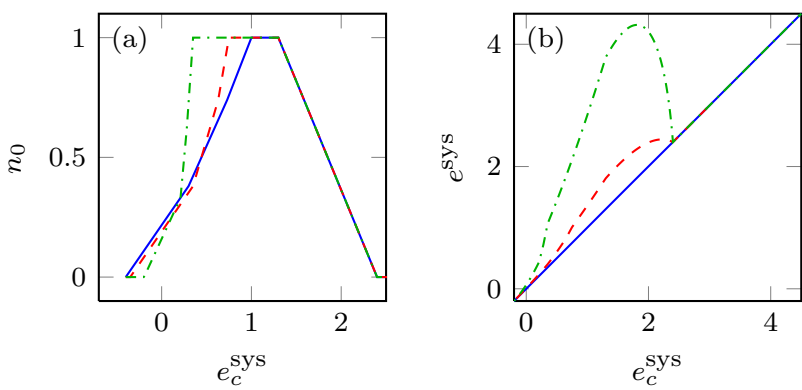

Fig. 6 Influence of $E s$ on the static calibration: blue solid line for infinite $E_{s}$; red dashed for $E_{s}=3.10^{5} \mathrm{~Pa}$; green dotdashed for $E_{s}=7.10^{4} \mathrm{~Pa}$

that the course of $e_{c}$ during contraction becomes larger for a given afterload, since we start from $e_{c}=e$ with the extension of the preload. Nevertheless, the calibration of $E_{s}$ would require specific data based on measurements of the sarcomere extensions for various controlled levels of active forces, see e.g. (Linari et al, 2004, 2007, 2009; Piazzesi et al, 2007). Since we do not have such data at hand in our case, we use a large value of $E_{s}$ in the rest of this study.

Calibration of activation. The kinetics of contraction strongly depend on the activation function $\bar{u}$ via $u$, see (4). As stated in the model presentation, this function represents a variable reaction rate, and in fact summarizes various complex chemical mechanisms that occur during depolarization and repolarization (Chapelle et al, 2012). In practice, we essentially need to prescribe in $u$ two nominal values characteristic of the time constants of active stress buildup and decay during these two stages, respectively - with a negative sign for repolarization by construction of our active law - with some transients ensuring continuous evolution throughout. This leads to a natural description of the function as piece-wise linear with two plateaus associated with the depolarization and repolarization rates. Note that of course alternative parametrizations of similar activation functions could be considered, e.g. with smoother exponential functions, albeit limited impact is to be expected in view of the above considerations on the activation function behavior. We then adjust this activation function so that the isometric stress curve corresponds to experimental measurements, see Fig.7 for the calibrated function. Note that, in particular, the point of maximum tip force must correspond to $u=0$, and subsequent negative values of $u$ induce an extension via a decrease of the active force. Moreover, the minimum (negative) value of $u$ is obtained from the slope of the stress relaxation curve in complete isometry for the large preload, choosing $m_{0}\left(e_{c}\right)=1$ in this case. We point out that the maximum (positive) value of $\bar{u}$ around $30 \mathrm{~s}^{-1}$ - the inverse of a time constant - is compatible with typical ATP turnover time scales (Lymn and Taylor, 1971; Linari et al, 2010). We further emphasize that the activation function $\bar{u}$ is calibrated once and for all with a single isometric force measurement, and then used as is in all subsequent simulations with varying preloads and afterloads. This activation function is thus representative of the electric state prevailing in the experimental setup, which is quite different from what occurs in rat hearts in vivo, in terms of time constants, in particular (heart rate at rest is about 300 bpm).

Load-dependent relaxation. Once the activation is calibrated, we adjust the parameters controlling the loaddependence of relaxation, namely, via the function $m_{0}\left(e_{c}\right)$, the time constant $\alpha_{r}$, and the parameter $\alpha$ controlling the velocity-dependent destruction of bridges. We point out that the isotonic extension phase necessarily corresponds to the late part of the plateau on the force curves, plateau which ends at the precise moment when the muscle tip re-establishes the contact with the support. Therefore, $m_{0}$ and $\alpha$ together govern the duration of isotonic extension depending on the afterload - via $m_{0}$ - and on the preload due to $\alpha$, since extension will be larger to reach the support corresponding to a large preload, see Fig.8. After that, in isometric relaxation the force relaxation rate is directly governed by $m_{0}$ alone. We set $\alpha_{r}$ to a rather large value, see Tab.1, hence $m_{0}$ essentially depends on the end-systolic strain during the whole relaxation, and then the above considerations allow to calibrate $m_{0}$ and $\alpha$ based on the data corresponding to different preloads and afterloads.
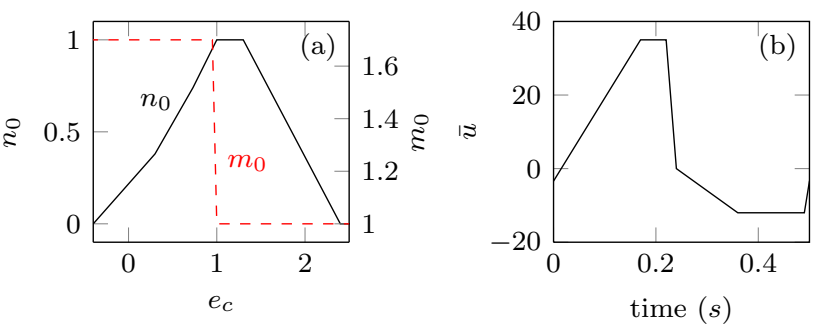

Fig. 7 (a) Length-dependence function $n_{0}$ (solid line) and load dependent relaxation $m_{0}$ (red dashed line); (b) activation function

\subsection{3 $1 D$ simulation results}

Fig.8 shows the dynamics of strain $e$ and tension $F_{\text {tip }} / A_{0}$, as simulated by the $1 \mathrm{D}$-model (solid lines) and compared to experimental data for the two different preloads (dashed lines), while Fig.9 shows the Hill-type 

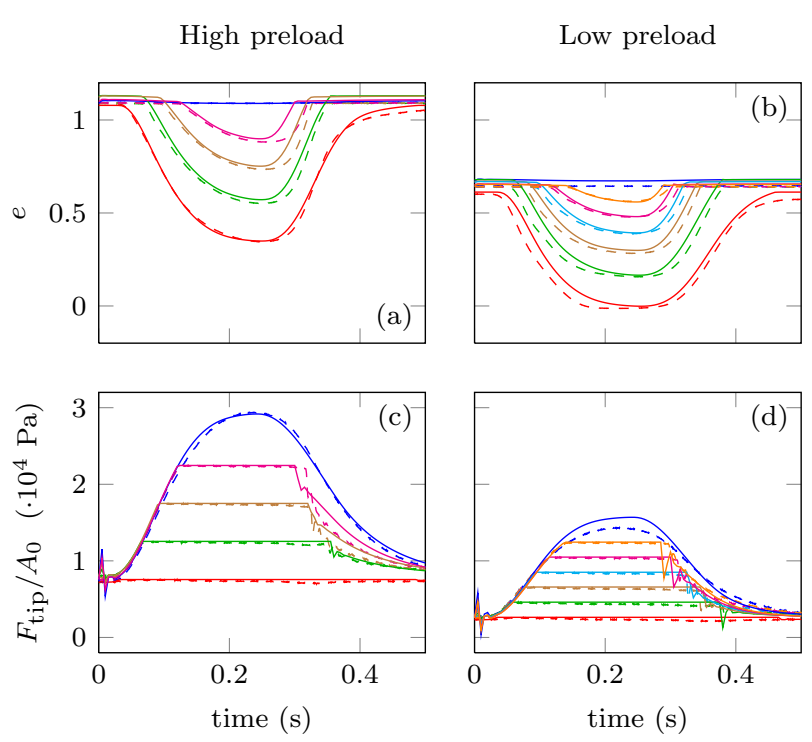

Fig. 8 Strain (top) and stress (bottom) response of a single papillary muscle for two different preloads. Dashed lines, experimental measurements; solid lines, simulations. Curves with the same color in each column correspond to the same afterload value

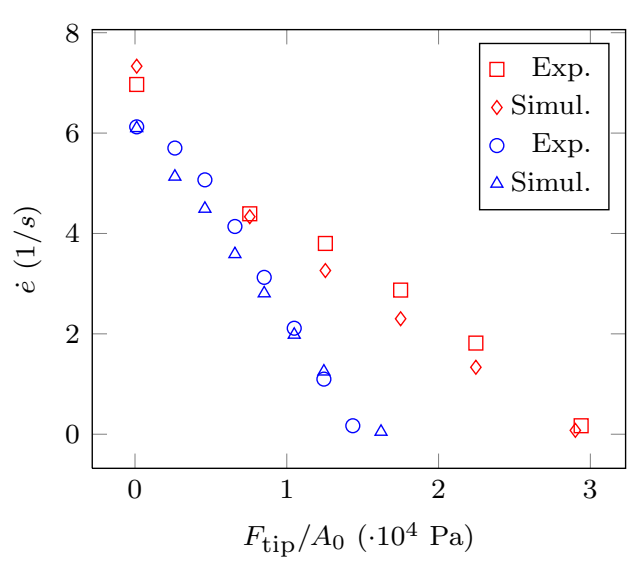

Fig. 9 Force-velocity plot from experimental data and numerical simulations - Low/high preload in blue/red

force-velocity curves obtained by taking the maximum velocity in the isotonic contraction phase.

We see that our 1D-model is able to most adequately reproduce the kinetics of the muscle sample response for the two preload values with the same set of parameters and the same activation function. This can be seen in the rise of the tensile force, in particular, the path of which mostly depends on the preload, which is well accounted for by the length-dependence function $n_{0}$ that induces a lower steady-state active force value for the smaller preload, and hence a smaller rate of increase.

Note that the observed small discrepancies in the initial values of strains - which is more visible for the lowest preload, see Fig.8 (b) - are the consequence of the large compliance of the passive stress-strain law for small strains, see Fig.5 (a), and small differences in the preload values used in the tests.

Regarding the force-velocity plot in Fig.9, although the simulated points lie on a rather "flat" curve - for each preload value - compared to the theoretical hyperbola predicted by A.V. Hill (Hill, 1938), we find an excellent match with our corresponding experimental data. In addition, these plots are consistent with the property of a unique intercept on the zero-force ordinate, namely, the $V_{0}$ maximum velocity of A.V. Hill, of about $6 \mathrm{~s}^{-1}$ here. Note that it is known that subtle tuning of the device damping would be required to obtain more convex force-velocity curves (Brutsaert and Claes, 1974), notwithstanding that departures from convexity have also been precisely characterized in experiments performed on myocardium (De Clerck et al, 1977; Daniels et al, 1984; de Tombe and ter Keurs, 1990). Nevertheless, this experimental tuning was not performed here, hence is not reproduced in the calibrated simulations either.

Our 1D-model also adequately represents the loaddependent relaxation, with the stress relaxation rate governed by $m_{0}$ and $\alpha$ as explained above.

\subsection{Application of the 0D-model}

Our objective in this section is to investigate to what extent the above-derived simple 0D model can reproduce, at least in an approximate manner, some important features of cardiac physiology. In this context, only qualitative or semi-quantitative correspondence is to be expected, and we will focus on physiological indicators representing the behavior of a healthy human heart. As regards parameter calibration, we will use the prior calibration of the $1 \mathrm{D}$ model as a starting point, albeit some recalibration of common parameters - as limited as possible, ideally - as well as further calibration of new parameters is required, due to differences between the two modeling contexts.

\subsubsection{Calibration strategy}

The atrial pressure is prescribed as a given function of time, calibrated using physiological values, in correspondence with the passive behavior calibration. We aim at obtaining a maximum volume around $120 \mathrm{~mL}$ including about $20 \%$ acquired during atrial contraction, and with an inflow twice as high - in terms of maximum volume increase rate - during the first stage of ventricular filling than during atrial contraction (Guyton and Hall, 2011). We check that these specifications can be fulfilled with realistic values of the atrial pressure while 
leaving the passive behavior parameters as calibrated with the 1D model and data.

The active behavior is recalibrated in order to obtain physiological end-systolic states, namely, values of pressure and volume - with adequate ejection fraction, in particular - and the slope of the locus of these endsystolic states in the pressure-volume diagram for varying afterload levels, i.e. the above-discussed ESPVR. To that purpose we only modify the contractility parameter $\sigma_{0}$, which is taken about one order of magnitude larger in the 0D case. We discuss some possible explanations for this difference in calibration below. We emphasize that the activation function $u$-including the relaxation modeling components in (3)-(4) - and the length-dependence $n_{0}$ function are left unchanged.

In addition, we here need to calibrate the circulation model. First the distal circulation parameters, $R_{d}$ and $C_{d}$, are chosen so that the characteristic time of the evolution of the distal pressure $P_{d}$ - with the time constant given by $R_{d} C_{d}$-is compatible with the cycle duration, i.e. $0.8 \mathrm{~s}$ here, while $C_{d}$ alone governs the distal pressure increase. Likewise, the proximal parameters $R_{p}$ and $C_{p}$ are adjusted so that the associated time constant is about $10^{-2} \mathrm{~s}$ and the proximal resistance $R_{p}$ conditions the peak ventricular pressure. The parameters of the valve law are chosen so that the pressure-flow relationship (12) is as close as possible to the ideal behavior without leading to numerical difficulties (Sainte-Marie et al, 2006).

The final values of the calibrated parameters are listed in Tab.1.

\subsubsection{Simulation results with the OD-model}

Typical cardiac cycle. In Fig.10, we plot the results obtained with the 0D-model. We find that our 0D-model produces a realistic contraction cycle representative of a normal human left ventricle, with - in particular - a physiological ejection fraction of about $60 \%$, and the maximum filling inflows (negative peaks in Fig.10 (b)) in the above-discussed proportion. As this simulation was obtained with a straightforward recalibration of the model validated using the papillary muscle experiments, this confirms the rather direct correspondence between local properties and a macroscopic organ model.

ESPVR assessment. The ESPRV is one of the most frequently used indicators in normal and pathological cardiac physiology to assess the cardiac condition. This relation has been shown to be independent of the preload and afterload (Gordon et al, 1966). We can obtain the corresponding pressure-volume curve by considering the
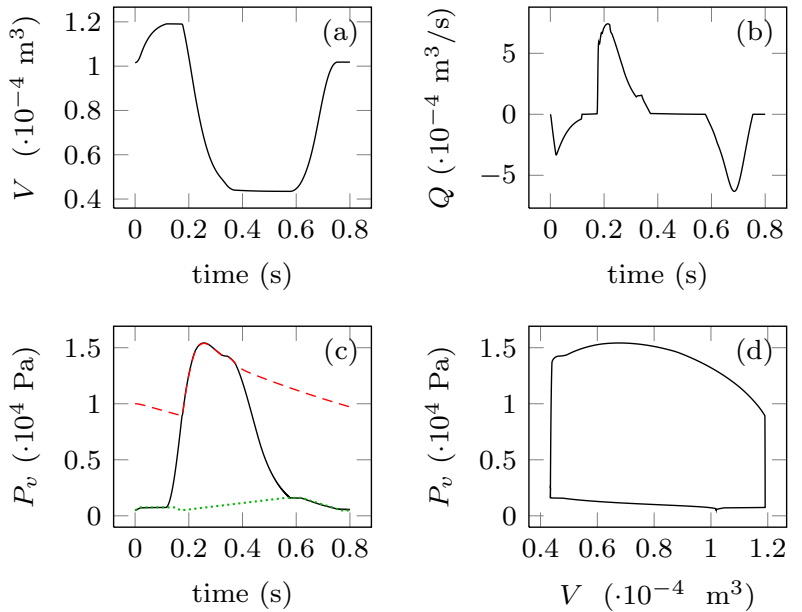

Fig. 10 Cardiac cycle obtained with the 0D-model: (a) left ventricular volume; (b) cardiac outflow (positive during systole); (c) ventricular (solid), proximal aortic (red, dashed), and atrial (green, dotted) pressures; (d) pressure-volume cycle

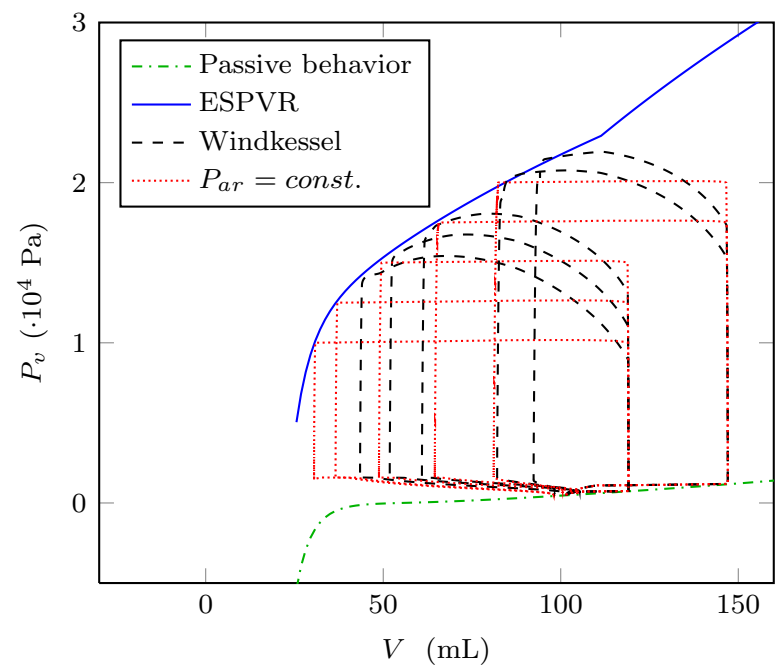

Fig. 11 Comparison between physiological loading (black, dashed) with ideal constant afterload condition (red, dotted); theoretical ESPVR in blue and passive behavior in dotdashed green

steady state of (19), namely,

$P_{v}=\frac{d_{0}}{R_{0}}\left(1+\frac{y}{R_{0}}\right)^{-1} \Sigma_{\mathrm{sph}}$

for different values of the volume, as visualized by the blue curve in Fig.11. Available experimental data usually lead to the ESPVR being well-approximated by a straight line, as is also seen in our figure in the physiological volume range, even if different shapes are also sometimes reported (Takeuchi et al, 1991; Senzaki et al, 1996). Regarding the characterization of the ESPVR by Eq.(24), we point out that, for physiological values of the end-systolic volumes, the corresponding strains are 
associated with very low passive stresses in this relation, hence, $\Sigma_{\mathrm{sph}}$ is then essentially the result of the active stress, itself governed by the $n_{0}$ function. Therefore, we find that the ESPVR curve is a rather direct image - at the global organ level - of the length-dependence mechanism occurring at the sarcomere level. Note also that the major features of the cardiac function as a biomechanical pump are then essentially conditioned by the two curves associated with passive behavior and ES$\mathrm{PVR}$, as seen in the figure.

As reported in earlier works, the end-systolic points lie on the ESPVR curve regardless of the ejection path, namely, independently of the external circulation system state (Suga et al, 1973). As an illustration of this phenomenon, we reproduce the constant afterload experiments of (Suga et al, 1973) by changing the parameters of the distal components of the Windkessel model, and we see in Fig.11 that the new end-systolic points also lie on the ESPVR curve, indeed.

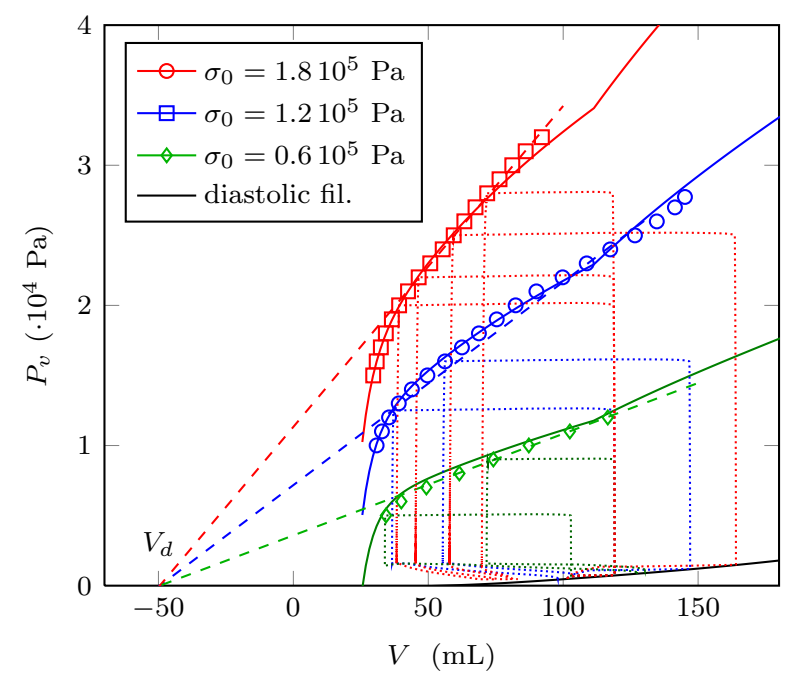

Fig. 12 ESPVR with different contractility values; symbols show the end-systolic points obtained from numerical simulations while solid lines give the theoretical ESPVR relations; dotted lines show different pressure-volume cycles under constant afterload for various preload and afterload conditions; solid black curve represents the passive behavior

In Fig.12, we show the ESPVR variations induced by changes in the contractility parameter, namely, $\sigma_{0}$, which corresponds to varying the inotropy. As is observed in cardiac physiology (Suga et al, 1973; Guyton and Hall, 2011), we obtain ESPVR curves of increasing slopes when the contractility increases - proportionally to $\sigma_{0}$ in our model - and with a common intercept on the volume axis, usually denoted by $V_{d}$.

Our results are thus fully compatible with the property that the ESPVR curve be independent of both preload and afterload. Preload-independence is - of course - directly associated with the Frank-Starling mecha- nism. Moreover, the ESPVR is in direct relation with the microscopic mechanical properties and in particular with the length-dependence of the maximum active force represented by our function $n_{0}$.
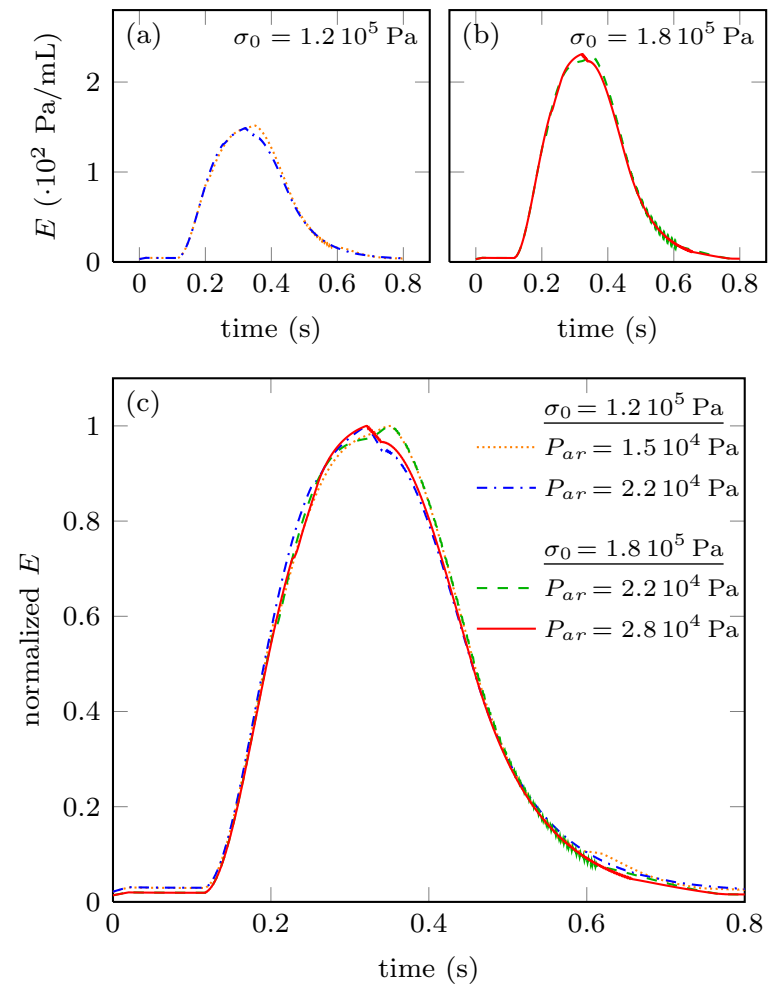

Fig. 13 Elastance recorded for two different values of contractility $\sigma_{0}=1.210^{5} \mathrm{~Pa}$ (a) and $\sigma_{0}=1.810^{5} \mathrm{~Pa}$ (b); Rescaled elastance curves for all contractility and afterload values $(c)$

Time-varying elastance. As in (Suga et al, 1973) we define the instantaneous ventricular elastance $E$ as

$E(t)=\frac{P_{v}(t)}{V(t)-V_{d}}$

and we represent in Fig.13 the evolution of this quantity in time under constant afterload. More specifically, we consider two different contractility values, with two different afterload levels for each of them. We see very little difference, for each contractility value, between the curves obtained for the two different afterload levels, as experimentally evidenced in (Suga et al, 1973; Iribe et al, 2006). Following the classical approach, we then normalize the elastance by its maximum value in time - for each simulation separately. When plotting all these curves together in Fig.13 (c), we obtain very similar kinetics between the various curves despite the variations of contractility and afterload, as experimentally observed in (Suga et al, 1973), indeed. 


\section{Discussion}

We have demonstrated how dimensional reductions of a 3D model can be performed in a consistent manner as regards the passive and active components of the $3 \mathrm{D}$ behavior. This approach has generic value, as we assumed no particular simplifying property of these constitutive ingredients in the process, and the main technicalities then reside in the elimination of the hydrostatic pressure based on the stress state properties, and the derivation of the strain invariants specialized to the kinematics at hand. In this respect, this extends some previous approaches in which similar reductions were performed under specific assumptions concerning the passive constitutive behavior, as in (Arts et al, 2003) where the only passive contribution in the directions transverse to muscle fibers is that of hydrostatic pressure, and likewise with the passive law used in (Hunter et al, 1998) when applied in the uniaxial tension case.

As mentioned when describing the simulations of the $1 \mathrm{D}$ model, the main limitation of this preliminary validation trial with experimental data concerns the range of strains found in correspondence with the data, clearly unrealistic at the sarcomere level. We believe that this is largely due to passive tissue in a rather large vicinity of the clips - as e.g. precisely evidenced in (Krueger and Pollack, 1975; ter Keurs et al, 1980) - and introducing some additional series-type contribution in the overall constitutive behavior. Characterizing this distinct contribution would require more detailed measurements, out of the scope of this paper. Therefore, our validation can only be considered as "semiquantitative", and the elucidation of the additional series contribution is bound to lead to a rescaling of the strain scale in the actual passive constitutive law of the myocardium, and likewise for the $n_{0}$ function representing the length-dependence. Nevertheless, our trials have shown that this function is adequate to represent the systolic load-dependence effects.

As regards the difference in calibration between the two reduced models, we recall that we only changed the contractility parameter, within all the physical parameters shared by the two models. It should be noted that in the $1 \mathrm{D}$ model the contractility parameter corresponds to the contractility of a rat papillary muscle in experimental conditions, whereas in the 0D model the required contractility is that of a human heart wall in physiological conditions. First of all, we do not expect the observed difference to arise from inter-species variability, since a rat heart is known to operate in very similar ranges of stresses and strains as the human heart, see (Pacher et al, 2008). Hence, the discrepancy may be attributed to functional changes in ex-vivo experimen- tal conditions, due to e.g. some kind of myocardial stunning (Kloner and Jennings, 2001), and to a perfusion state different from physiological conditions since we recall that in the experimental setup perfusion is provided by the solution in which the sample is immersed. In addition, there may be differences in active contents between papillary muscles and wall tissue. Such differences may be experimentally assessed by measurements pertaining to myosin contents as performed in (Fitzsimons et al, 1998), but we do not know of any reference providing such comparative measurements for both the papillary muscles and the wall tissue in the same organ. At any rate, in our case the maximum measured active stress - directly related to our contractility parameter - appears to be within the range of other such experimentally measured values available in the literature in similar protocols, see in particular a survey in (Niederer et al, 2006). In the latter study, a quite large variability - by a factor 5 , typically, between largest and lowest values - is revealed in such measured data, and our calibrated value is in fact in the lowest part of the experimental range. Nevertheless, effective contractility being clearly proportional to the density of recruitable active elements, it is natural to take into account this density and the deficit thereof - whether it may result from structural factors or experimental conditions - to directly scale the contractility parameter of the model to account for such different conditions. The fact that only this parameter needs to be modified in our $0 \mathrm{D}$ calibration is in line with this conjecture concerning possible variations in active contents, indeed.

Through the use of our two reduced-dimensional modeling strategies, we have shown that our cardiac tissue model is fully compatible with the ESPVR concept. Our findings also confirm the direct relation of the ESPVR with the sarcomere scale properties of lengthdependent maximum active force, represented in our case by the function $n_{0}$ that has a direct image in the pressure-volume space at the organ level as substantiated by our 0D-model. This could be further exploited in many ways. For instance, we could test the effects of drugs on papillary muscles to emulate some cardiac diseases, accordingly recalibrate (or estimate) the $n_{0^{-}}$ function and all constitutive parameters based on the 1D-model, and then use the 0D-model to assess the impact on a cardiac cycle.

The ESPVR is frequently discussed in relation with the Frank-Starling effect, namely, the increase of stroke volume for increasing preloads. Here, we see that in our model the two properties are - in essence - accurately represented by the same $n_{0}$ function, indeed. In addition, in our model we see that this function has a direct impact on the rate of active force increase during 
contraction. This suggests an additional interpretation of the Frank-Starling effect, namely, that it primarily works by increasing this active force build-up rate to allow a larger strain variation in the same time interval - hence, an increased contraction velocity - for increased end-diastolic strains associated with a higher preload. This is also fully consistent with the Hill type force-velocity relations, of course.

Concerning the diastolic load-dependence phenomena, we have proposed a simple modeling ingredient with a variable following a first-order dynamics that is used to weigh the activation function, to account for the variations of unbinding kinetics due to steric effects in the sarcomere. We have found that this - combined with the strain rate unbinding term associated with the parameter $\alpha$ - allows to reproduce quite accurately the effects measured in the experimental data. However, we do not claim that the same level of validation has been achieved here as for systolic effects, since diastolic effects are much more subtle to characterize based on experimental data. Furthermore, such effects intervene in a different manner in actual cardiac physiology, in which diastole starts with an isovolumic phase, as opposed to isometric in the experimental protocol - recall Fig.5 (a) where only the two orthogonal segments 1-2-3 are explored in both directions instead of a full cycle. This means that detailed measurements would also be required to investigate this issue, and possibly to reconcile these effects with the variable elastance theory, or to identify in which specific context the effects in question may represent a departure from this theory.

\section{Conclusion}

We have proposed a generic approach for deriving reduced-dimensional versions of a $3 \mathrm{D}$ heart model. The 1D model was intended to accurately represent the behavior of elongated structures such as muscle samples or myocytes, and we achieved a detailed validation of our model based on experimental data produced with papillary muscles, in particular concerning length-dependence effects in both systole and diastole. The 0D model was designed as an approximate representation of a cardiac cavity - typically the left ventricle - and we have obtained some most adequate pressure-volume indicators when running simulations of this model with the parameter values pre-calibrated with the $1 \mathrm{D}$ model results, including when assessing the ESPVR and varying elastance properties. The 0D model can also readily be used as a calibration tool for detailed 3D model simulations, or as a boundary condition to an arterial model, whether to prescribe the inflow, or both fluid and solid input conditions when appropriate, see (Moireau et al, 2012).

Moreover, our approach of devising several models within a single hierarchical family sheds some new light on the relations between the phenomena observed at different scales and in different contexts. As could be expected, we find a direct translation of the lengthdependence effect modeled at the sarcomere level and the load-dependent contraction of a papillary muscle modeled in our 1D framework. Still more interestingly, considering concurrently the 1D and OD models allows to directly translate at the organ level some findings obtained at the local level of the sample, and in particular we have found that the ESPVR curve is a straightforward image in the cardiac function of the lengthdependence effects observed at the lower scales. More generally, this modeling framework could be used as a tool for predicting the in-vivo manifestations in the heart of phenomena observed - or induced - in vitro in various types of experimental testing, such as for assessing the effects of drugs.

Further perspectives include detailed assessments of a series elasticity contribution in the myocardium constitutive behavior, based on specific data to be obtained. Moreover, estimation methodologies - also known as data assimilation, see (Chapelle et al, 2013) and references therein - can be expected to be particularly effective in reduced-dimensional models to automatically identify model parameters. Indeed, estimation typically leads to very intensive computations in $3 \mathrm{D}$ models, and convergence in the estimation process is dependent on the parameter values used as starting points, hence a preliminary stage of estimation based on a reduced model would be of substantial potential benefit.

\section{References}

Arts T, Bovendeerd PH, Prinzen FW, Reneman RS (1991) Relation between left ventricular cavity pressure and volume and systolic fiber stress and strain in the wall. Biophys J 59(1):93-102

Arts T, Bovendeerd P, Delhaas T, Prinzen F (2003) Modeling the relation between cardiac pump function and myofiber mechanics. Journal Of Biomechanics 36(5):731-736

Bathe KJ (1996) Finite Element Procedures. Prentice Hall, Englewood Cliffs

Bestel J, Clément F, Sorine M (2001) A biomechanical model of muscle contraction. In: Medical Image Computing and Computer-Assisted Intervention - MICCAI 2001, Springer, pp 1159-1161 
Bluhm WF, McCulloch AD, Lew WYW (1995) Active force in rabbit ventricular myocytes. J Biomechanics 28(9):1119-1122

Brutsaert DL, Claes VA (1974) Onset of mechanical activation of mammalian heart muscle in calcium- and strontium-containing solutions. Circ Res 35(3):345357

Brutsaert DL, Housmans PR, Goethals MA (1980) Dual control of relaxation. Its role in the ventricular function in the mammalian heart. Circ Res 47(5):637-652

Campbell KS (2011) Impact of myocyte strain on cardiac myofilament activation. Pflug Arch Eur J Phy 462(1):3-14

Cazorla O, Le Guennec JY, White E (2000) Lengthtension relationships of sub-epicardial and subendocardial single ventricular myocytes from rat and ferret hearts. J Mol Cell Cardiol 32(5):735-744

Chabiniok R, Moireau P, Lesault PF, Rahmouni A, Deux JF, Chapelle D (2011) Estimation of tissue contractility from cardiac cine-MRI using a biomechanical heart model. Biomech Model Mechanobiol 11(5):609-630

Chapelle D, Bathe KJ (2011) The Finite Element Analysis of Shells - Fundamentals, 2nd edn. Springer

Chapelle D, Le Tallec P, Moireau P, Sorine M (2012) An energy-preserving muscle tissue model: formulation and compatible discretizations. Int J Multiscale Com 10(2):189-211

Chapelle D, Fragu M, Mallet V, Moireau P (2013) Fundamental principles of data assimilation underlying the Verdandi library: applications to biophysical model personalization within euHeart. Med Biol Eng Comput DOI 10.1007/s11517-012-0969-6

Claes VA, Brutsaert DL (1971) Infrared-emitting diode and optic fibers for underwater force measurement in heart muscle. J Appl Physiol 31(3):497-498

Costa K, Holmes J, McCulloch A (2001) Modelling cardiac mechanical properties in three dimensions. Philos T Roy Soc A 359(1783):1233-1250

Daniels M, Noble MI, ter Keurs HE, Wohlfart B (1984) Velocity of sarcomere shortening in rat cardiac muscle: relationship to force, sarcomere length, calcium and time. J Physiol 355:367-381

De Clerck NM, Claes VA, Brutsaert DL (1977) Force velocity relations of single cardiac muscle cells: calcium dependency. J Gen Physiol 69(2):221-241

Fabiato A, Fabiato F (1975) Dependence of the contractile activation of skinned cardiac cells on the sarcomere length. Nature 256(5512):54-56

Fitzsimons DP, Patel JR, Moss RL (1998) Role of myosin heavy chain composition in kinetics of force development and relaxation in rat myocardium. $\mathrm{J}$
Physiol 513(1):171-183, DOI 10.1111/j.1469-7793. 1998.171by.x, URL http://dx.doi.org/10.1111/ j.1469-7793.1998.171by.x

Frank O (1895) Zur Dynamik des Herzmuskels. Z Biol 32:370-447

Fukuda N, Sasaki D, Ishiwata S, Kurihara S (2001) Length dependence of tension generation in rat skinned cardiac muscle - Role of titin in the Frank-Starling mechanism of the heart. Circulation 104(14):1639-1645

Gordon AM, Huxley AF, Julian FJ (1966) The variation in isometric tension with sarcomere length in vertebrate muscle fibres. J Physiol 184(1):170-192

Guerin T, Prost J, Joanny JF (2011) Dynamical behavior of molecular motor assemblies in the rigid and crossbridge models. Eur Phys J 34(6):60

Guyton AC, Hall JE (2011) Textbook of Medical Physiology, 12th edn. Elsevier

Hill AV (1938) The heat of shortening and the dynamic constants of muscle. P Roy Soc Lond B Bio 126(843):136-195

Holzapfel GA, Ogden RW (2009) Constitutive modelling of passive myocardium: a structurally based framework for material characterization. Philos T Roy Soc A 367(1902):3445-3475

Hunter PJ, McCulloch AD, ter Keurs HEDJ (1998) Modelling the mechanical properties of cardiac muscle. Prog Biophys Molec Biol 69(2-3):289-331

Huxley AF (1957) Muscle structure and theories of contraction. Prog Biophys Molec Biol 7:258-318

Iribe G, Helmes M, Kohl P (2006) Force-length relations in isolated intact cardiomyocytes subjected to dynamic changes in mechanical load. Am J PhysiolHeart Circ Physiol 292(3):H1487-H1497

Izakov VY, Katsnelson LB, Blyakhman FA, Markhasin VS, Shklyar TF (1991) Cooperative effects due to calcium binding by troponin and their consequences for contraction and relaxation of cardiac muscle under various conditions of mechanical loading. Circ Res 69(5):1171-1184

Julian FJ, Sollins MR (1975) Sarcomere length-tension relations in living rat papillary muscle. Circ Res 37(3):299-308

Kentish JC, ter Keurs HE, Ricciardi L, Bucx JJ, Noble MI (1986) Comparison between the sarcomere length-force relations of intact and skinned trabeculae from rat right ventricle. Influence of calcium concentrations on these relations. Circ Res 58(6):755768

Kerckhoffs RCP, Faris OP, Bovendeerd PHM, Prinzen FW, Smits K, McVeigh ER, Arts T (2005) Electromechanics of paced left ventricle simulated by straightforward mathematical model: comparison 
with experiments. Am J Physiol Heart Circ Physiol 289(5):H1889-97

ter Keurs HE, Rijnsburger WH, van Heuningen R, Nagelsmit MJ (1980) Tension development and sarcomere length in rat cardiac trabeculae. Evidence of length-dependent activation. Circ Res 46(5):703-714

Kloner R, Jennings R (2001) Consequences of brief ischemia: Stunning, preconditioning, and their clinical implications, part 1. Circulation 24(104):2981-2989

Koiter W (1965) On the nonlinear theory of thin elastic shells. Proc Kon Ned Akad Wetensch B69:1-54

Krueger JW, Pollack GH (1975) Myocardial sarcomere dynamics during isometric contraction. J Physiol 251(3):627-643

Lecarpentier YC, Chuck LH, Housmans PR, De Clerck NM, Brutsaert DL (1979) Nature of load dependence of relaxation in cardiac muscle. Am J Physiol-Heart Circ Physiol 237(4):H455-H460

Linari M, Bottinelli R, Pellegrino M, Reconditi M, Reggiani C, Lombardi V (2004) The mechanism of the force response to stretch in human skinned muscle fibres with different myosin isoforms (vol, 554, pg 335, 2004). J Physiol 555:851

Linari M, Caremani M, Piperio C, Brandt P, Lombardi V (2007) Stiffness and fraction of myosin motors responsible for active force in permeabilized muscle fibers from rabbit psoas. Biophys J 92:2476-2490

Linari M, Piazzesi G, Lombardi V (2009) The effect of myofilament compliance on kinetics of force generation by myosin motors in muscle. Biophys $\mathrm{J}$ 96(2):583-592

Linari M, Caremani M, Lombardi V (2010) A kinetic model that explains the effect of inorganic phosphate on the mechanics and energetics of isometric contraction of fast skeletal muscle. P Roy Soc Lond B Bio 277(270):19-27

Linke W, Fernandez J (2002) Cardiac titin: molecular basis of elasticity and cellular contribution to elastic and viscous stiffness components in myocardium. J Muscle Res Cell Motil 23(5-6):483-497

Linke WA, Popov VI, Pollack GH (1994) Passive and active tension in single cardiac myofibrils. Biophys J $67(2): 782-792$

Lumens J, Delhaas T, Kirn B, Arts T (2009) Threewall segment (TriSeg) model describing mechanics and hemodynamics of ventricular interaction. Ann Biomed Eng 37(11):2234-2255

Lymn R, Taylor E (1971) Mechanism of adenosine triphosphate hydrolysis by actomyosin. Biochemistry 10(25):4617-\{\&

Moireau P, Chapelle D, Le Tallec P (2008) Joint state and parameter estimation for distributed mechanical systems. Computer Methods in Applied Mechanics and Engineering 197:659-677

Moireau P, Xiao N, Astorino M, Figueroa CA, Chapelle D, Taylor C A, Gerbeau JF (2012) External tissue support and fluid-structure simulation in blood flows. Biomech Model Mechanobiol 11(1-2):1-18, DOI 10. 1007/s10237-011-0289-z, URL http://hal.inria. fr/hal-00701801

Moss RL, Fitzsimons DP (2002) Frank-Starling Relationship - Long on importance, short on mechanism. Circ Res 90(1):11-13

Nash M, Hunter P (2000) Computational mechanics of the heart - From tissue structure to ventricular function. J Elasticity 61(1-3):113-141

Niederer SA, Smith NP (2009) The Role of the Frank-Starling Law in the Transduction of Cellular Work to Whole Organ Pump Function: A Computational Modeling Analysis. PLoS Comput Biol 5(4):e1000,371

Niederer SA, Hunter PJ, Smith NP (2006) A quantitative analysis of cardiac myocyte relaxation: A simulation study. Biophys J 90(5):1697-1722

Pacher P, Nagayama T, Mukhopadhyay P, Bátkai S, Kass DA (2008) Measurement of cardiac function using pressure-volume conductance catheter technique in mice and rats. Nature Protocols 3(9):1422-1434

Panerai RB (1980) A model of cardiac muscle mechanics and energetics. J Biomechanics 13:929-940

Parikh SS, Zou SZ, Tung L (1993) Contraction and relaxation of isolated cardiac myocytes of the frog under varying mechanical loads. Circ Res 72(2):297-311

Peskin CS (1975) Mathematical Aspects of Heart Physiology. Courant Institute of Mathematical Sciences

Piazzesi G, Reconditi M, Linari M, Lucii L, Bianco P, Brunello E, Decostre V, Stewart A, Gore DB, Irving TC, Irving M, Lombardi V (2007) Skeletal muscle performance determined by modulation of number of myosin motors rather than motor force or stroke size. Cell 131(4):784-795

Sachse FB (2004) Computational Cardiology: Modeling of Anatomy, Electrophysiology, and Mechanics. Springer

Sainte-Marie J, Chapelle D, Cimrman R, Sorine M (2006) Modeling and estimation of the cardiac electromechanical activity. Comput Struct 84(28):17431759

Schmid H, O'Callaghan P, Nash MP, Lin W, LeGrice IJ, Smaill BH, Young AA, Hunter PJ (2008) Myocardial material parameter estimation. Biomech Model Mechanobiol 7(3):161-173

Senzaki H, Chen CH, Kass DA (1996) Single-beat estimation of end-systolic pressure-volume relation in humans: a new method with the potential for noninvasive application. Circulation 94:2497-2506 
Shiels HA, White E (2008) The Frank-Starling mechanism in vertebrate cardiac myocytes. J Exp Biol 211(13):2005-2013

Smith N, et al (2011) euHeart: personalized and integrated cardiac care using patient-specific cardiovascular modelling. Interface Focus 1(3):349-364

Sonnenblick EH (1962) Force-velocity relations in mammalian heart muscle. Am J Physiol 202(5):931-939

Starling EH (1918) The Linacre Lecture on the Law of the Heart Given at Cambridge, 1915. Nature 101(2525):43-43

Suga H, Sagawa K, Shoukas AA (1973) Load independence of the instantaneous pressure-volume ratio of the canine left ventricle and effects of epinephrine and heart rate on the ratio. Circ Res 32(3):314-322

Takeuchi M, Igarashi Y, Tomimoto S, Odake M, Hayashi T, Tsukamoto T, Hata K, Takaoka H, Fukuzaki H (1991) Single-beat estimation of the slope of the end-systolic pressure-volume relation in the human left ventricle. Circulation 83:202-212

de Tombe PP, ter Keurs HE (1990) Force and velocity of sarcomere shortening in trabeculae from rat heart. Effects of temperature. Circ Res 66(5):1239-1254

Tortora GJ, Derrikson B (2009) Principles of Anatomy and Physiology, 12th edn. John Wiley \& Sons, Inc

Trayanova NA, Rice JJ (2011) Cardiac electromechanical models: from cell to organ. Front Physiol 2:43

Weiwad WK, Linke WA, Wussling MH (2000) Sarcomere length-tension relationship of rat cardiac myocytes at lengths greater than optimum. J Mol Cell Cardiol 32(2):247-259

Wong AYK (1972) Mechanics of cardiac muscle, based on Huxley's model: Simulation of active state and force-velocity relation. J Biomechanics 5(1):107-117

Zahalak GI, Ma S (1990) Muscle activation and contraction: constitutive relations based directly on cross-bridge kinetics. J Biomech Eng 112(1):52-62 